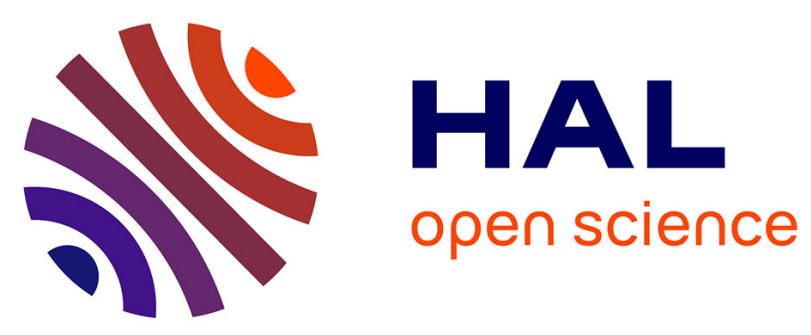

\title{
La chapelle Saint-Thomas d'Aizier (Eure) : bilan de douze années de fouille programmée
}

Cécile Chapelain de Seréville-Niel, Marie-Cécile Truc, Thibault Cardon, Thomas Guérin, Fabien Le Roux, Bruno Penna, Françoise Yvernaut

\section{To cite this version:}

Cécile Chapelain de Seréville-Niel, Marie-Cécile Truc, Thibault Cardon, Thomas Guérin, Fabien Le Roux, et al.. La chapelle Saint-Thomas d'Aizier (Eure) : bilan de douze années de fouille programmée. Lequoy, Marie-Clotilde. Journées archéologiques de Haute-Normandie: Évreux, 6-8 mai 2011, Publications des universités de Rouen et du Havre, pp.241-260, 2012, Haute-Normandie archéologique, 978-2-87775-559-7. halshs-01715451

\section{HAL Id: halshs-01715451 \\ https://shs.hal.science/halshs-01715451}

Submitted on 22 Feb 2018

HAL is a multi-disciplinary open access archive for the deposit and dissemination of scientific research documents, whether they are published or not. The documents may come from teaching and research institutions in France or abroad, or from public or private research centers.
L'archive ouverte pluridisciplinaire HAL, est destinée au dépôt et à la diffusion de documents scientifiques de niveau recherche, publiés ou non, émanant des établissements d'enseignement et de recherche français ou étrangers, des laboratoires publics ou privés. 


\title{
JOURNÉES ARCHÉOLOGIQUES de Haute-Normandie
}

\author{
Évreux, 6-8 MAI 2OI I
}




\section{La chapelle Saint-Thomas d'Aizier (Eure) : bilan de douze années de fouille programmée ${ }^{1}$}

Le site de la " chapelle Saint-Thomas » à Aizier fut une léproserie médiévale, puis un prieuré moderne dépendant de l'abbaye de Fécamp. Cet établissement a fait l'objet d'une fouille programmée durant 10 ans. La phase terrain est à présent achevée, mais les études sont toujours en cours. Cependant commence déjà à se dessiner le cadre de vie d'une petite communauté de lépreux, sans doute fort pauvre, qui vécut et mourut à Aizier entre les $\mathrm{XII}^{\mathrm{e}}$ et XVI ${ }^{\mathrm{e}}$ siècles.

\section{The Saint Thomas Chapel in Aizier: assessment of a twelve-year long excavation programme}

The site on which the Saint Thomas Chapel is located in Aizier was a medieval leprosarium, then a modern priory belonging to the Fécamp Abbaye. This establishment was the focus of a ten-year long excavation programme. The field work has now been completed. However, the research is still in progress. The living conditions of a small community of lepers, undoubtedly quite poor, who lived and died in Aizier between the 12th and 16th centuries, have already begun to emerge.

MOYEN ÂGE, LÈPRE, LÉPROSERIE, CHAPELLE, CIMETIÈRE.

Cécile NiEL, Marie-Cécile TRUC, avec la collaboration de Thibault CARdon, Thomas GuÉRIN, Fabien Le Roux, Bruno Penna † et Françoise Yvernault
L'étude des léproseries reste un domaine de recherche récent et les premiers travaux réalisés sur ce thème ont été menés à partir d'analyses principalement historiques et paléopathologiques. Les historiens s'intéressent en effet depuis plusieurs années à l'histoire des léproseries. Si un gros travail de dépouillement et de cartographie des informations relatives aux léproseries et aux lépreux d'après les sources textuelles a pu être réalisé par leur intermédiaire, ils ont aussi contribué à renouveler les connaissances sur l'environnement social ou matériel des édifices hospitaliers, mais aussi sur l'histoire des mentalités et de la charité. En revanche, l'aspect purement architectural des édifices constituant une léproserie est souvent délaissé, les vestiges étant parfois à peine évoqués ou décrits. La quasi absence de sources sur le sujet explique en grande partie ce silence, d'autant plus que les textes mentionnent rarement cet aspect. Depuis quelques années, des fouilles ont permis de renouveler les questionnements sur les édifices hospitaliers et leur environnement en les abordant directement par l'étude de la maladie, et notamment la lèpre, sous un angle à la fois historique, anthropologique et archéologique. En France, le nombre de sites fouillés est restreint et ceux qui sont intégralement étudiés sont encore moins nombreux. En effet, les recherches portent soit sur le cimetière, soit sur la chapelle, mais plus rarement sur les bâtiments de vie et encore moins souvent sur la globalité d'un site.

Les ruines romantiques d'une chapelle romane dédicacée à Thomas Becket se dressent en forêt de Brotonne, à $800 \mathrm{~m}$ au sud-est du village d'Aizier (fig. 1). Il s'agit du seul témoin encore visible de ce qui fut jadis une léproserie dépendant de l'abbaye de Fécamp (Seine-Maritime).

Implanté à flanc de coteau à une altitude moyenne de 76 m NGF, l'établissement domine la basse vallée de la Seine. Il est installé sur la frange septentrionale du plateau du Roumois qui est constitué d'un substratum calcaire secondaire (jurassique et crétacé), recouvert d'un manteau d'argile résiduelle à silex.

En 1998, en préalable à la restauration de la chapelle et à une mise en valeur du lieu, des sondages sont réalisés par le Groupe archéologique du Val de Seine (GAVS). Ils révèlent la présence de bâtiments, d'un cimetière et d'une voie. Les vestiges sont bien conservés et la stratigraphie semble montrer l'absence de réutilisation et de réoccupation du site, chose plutôt rare pour une léproserie. Ces résultats laissent entrevoir la possibilité d'étudier de manière complète un type d'établissement surtout connu au travers d'études textuelles (par exemple : Bériac, 1988 ; Tabuteau, 1996 ; Touati, 1996 ; etc.) et de façon beaucoup plus lacunaire

1 Cet article se veut le complément d'un premier état des fouilles publié en 2007 (Truc, Niel et al., 2007). 


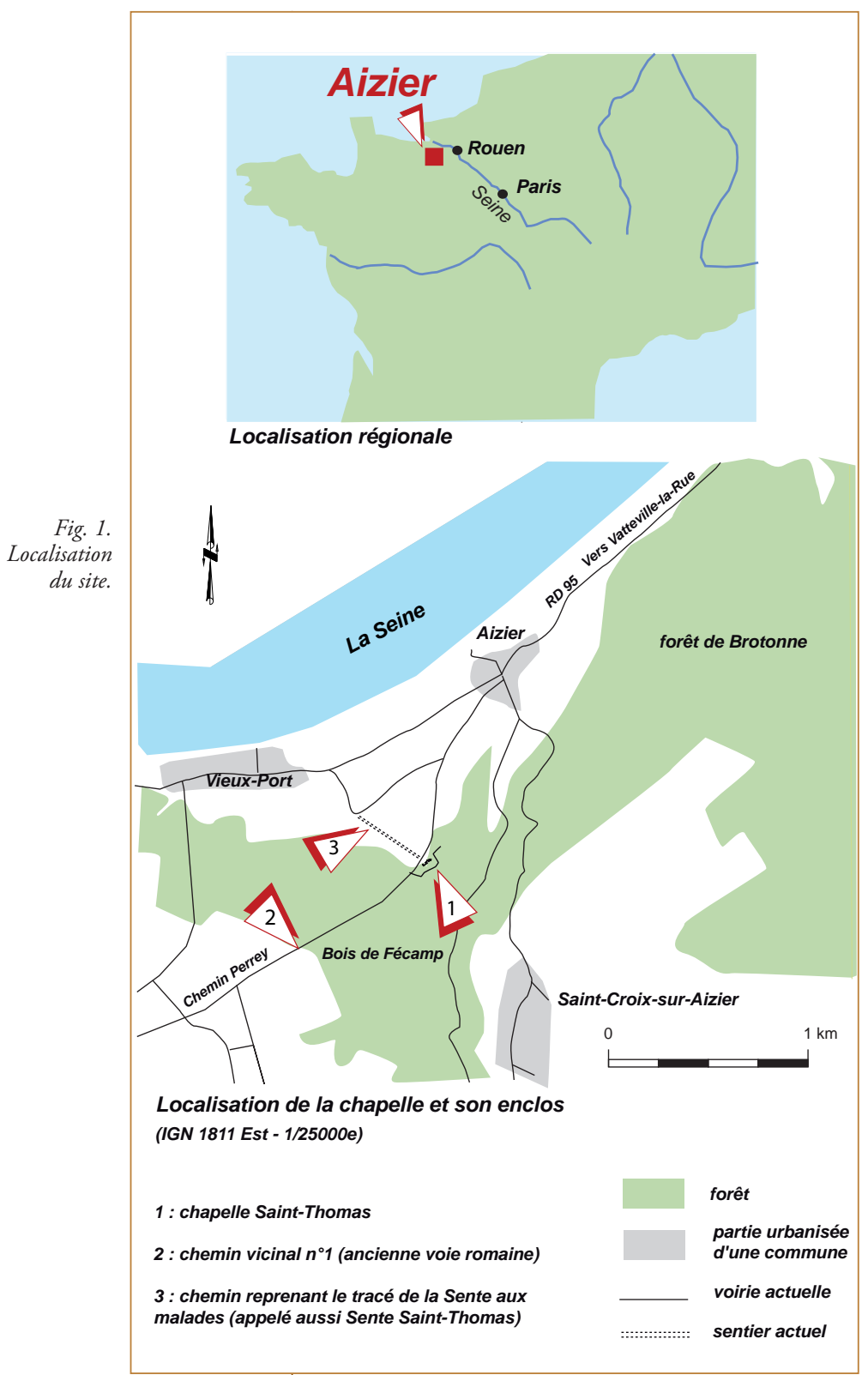

par l'archéologie. Les opérations menées dans ce dernier domaine ne concernent en général jamais l'intégralité du site, mais portent uniquement sur des secteurs bien précis (la chapelle ou le cimetière de préférence). Leur publication est par ailleurs encore très rare.

Le site fait l'objet d'une fouille programmée de 1998 à 2010. L'opération, réalisée en partenariat entre le GAVS et le CRAHAM, est conduite par Marie-Cécile Truc de 1998 à 2008, puis de 2009 à 2010 par Cécile Niel, qui assure également la responsabilité du secteur funéraire depuis 2005. Les fouilles ont permis l'exploration intégrale de la chapelle, la découverte de trois bâtiments à l'ouest de celle-ci, d'un cimetière au nord et de diverses structures au sud. Le reste de la parcelle est émaillé de fossés et talus parcellaire ainsi que de sentiers (fig. 2). Si la phase terrain est maintenant achevée, les études sont encore en cours. Elles sont réalisées dans l'objectif d'une publication monographique.

\section{Le monde des vivants : espaces bâtis et lieux de vie}

\section{La léproserie (fin du XII - milieu du XVI ${ }^{e}$ siècle ${ }^{2}$ )}

La terre d'Aizier est possession de l'abbaye de Fécamp depuis 1006 (Fauroux, 1961, p. 79-80). La date de fondation de la léproserie n'est pas connue, mais sa première mention remonte à $1227^{3}$. Les recherches archéologiques et architecturales permettent toutefois de situer une probable fondation vers la fin $\mathrm{du} \mathrm{XII}^{\mathrm{e}}$ siècle, époque du concile de Latran III et de la grande vague de fondation des léproseries (Tabuteau, 1995, t. 1, p. 98).

Les sources sont peu prolixes quant au statut de l'établissement, le nombre d'occupants ou encore les aspects de la vie quotidienne. En revanche, elles nous indiquent que Saint-Thomas est bien jusque dans le courant du Xvi ${ }^{\mathrm{e}}$ siècle une léproserie, dont l'administrateur est le curé d'Aizier qui réside au village. Ces derniers éléments plaident en faveur d'une léproserie modeste et de faible importance. Vers la fin du $\mathrm{XII}^{\mathrm{e}}$ siècle, est édifiée une chapelle constituée d'une nef rectangulaire de 13,4 m x $7 \mathrm{~m}$ et d'un chœur également rectangulaire mais plus étroit (6,8 m x $6 \mathrm{~m}$ ) (fig. 3). Le chevet plat est percé de deux baies romanes en plein cintre (fig. 4). L'entrée se faisait par le côté nord de la nef, comme en témoigne une grande pierre de seuil en calcaire toujours conservée.

Les murs, épais de $80 \mathrm{~cm}$, présentent un parement de silex équarris maçonnés enserrant un blocage interne de mortier, moellons de silex et craie. Ils reposent sur une fondation débordante de silex

2 La chronologie avancée dans cet article est provisoire, car l'étude céramique est en cours.

3 "Unam lampadam ardentam ante altare sanctis Thome annuatim " (Archives départementales de Seine-Martime [ADSM], 9H1275). 


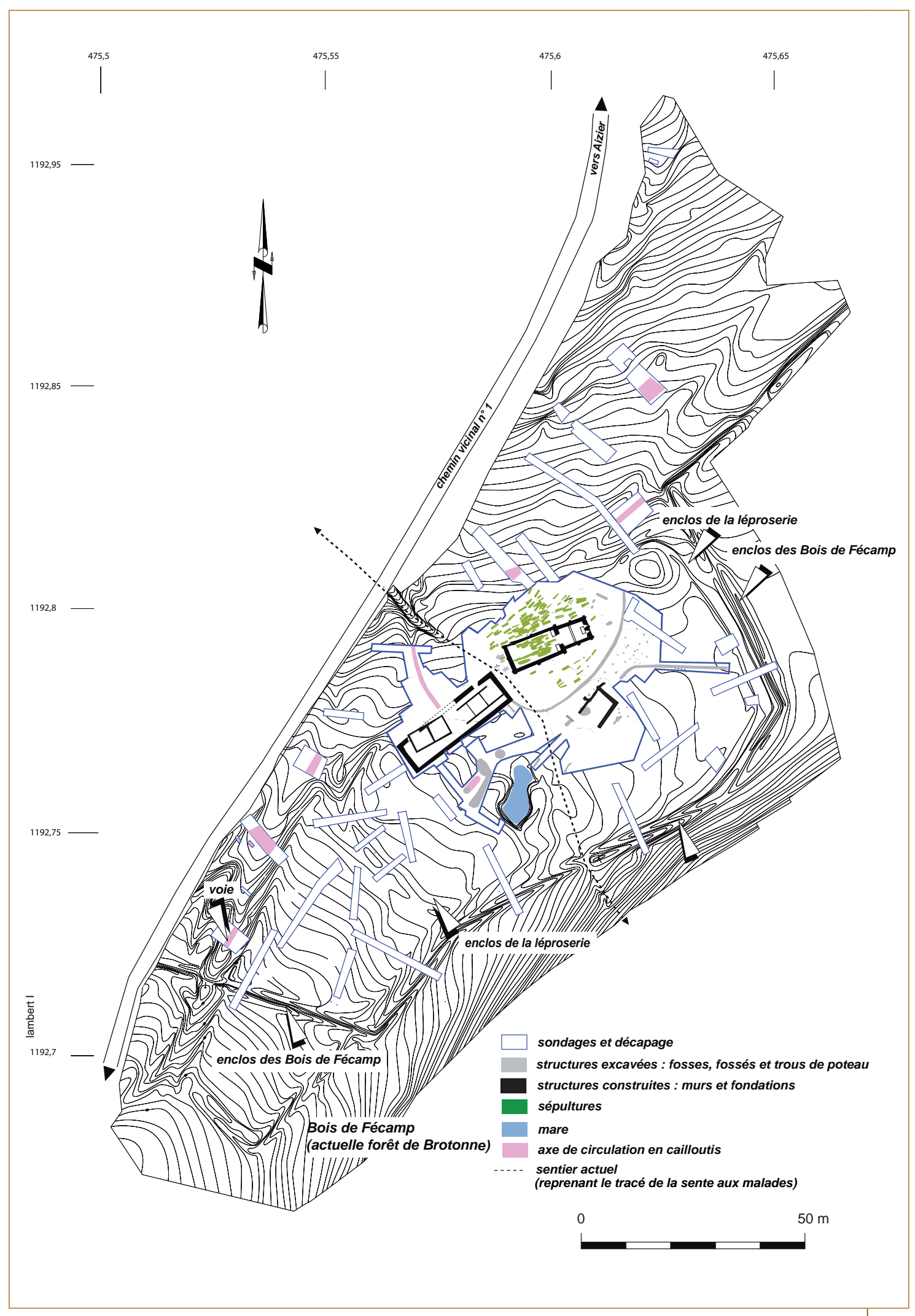

Fig. 2. Plan général du site (topographie et DAO:T. Guérin, M.-C. Truc). 


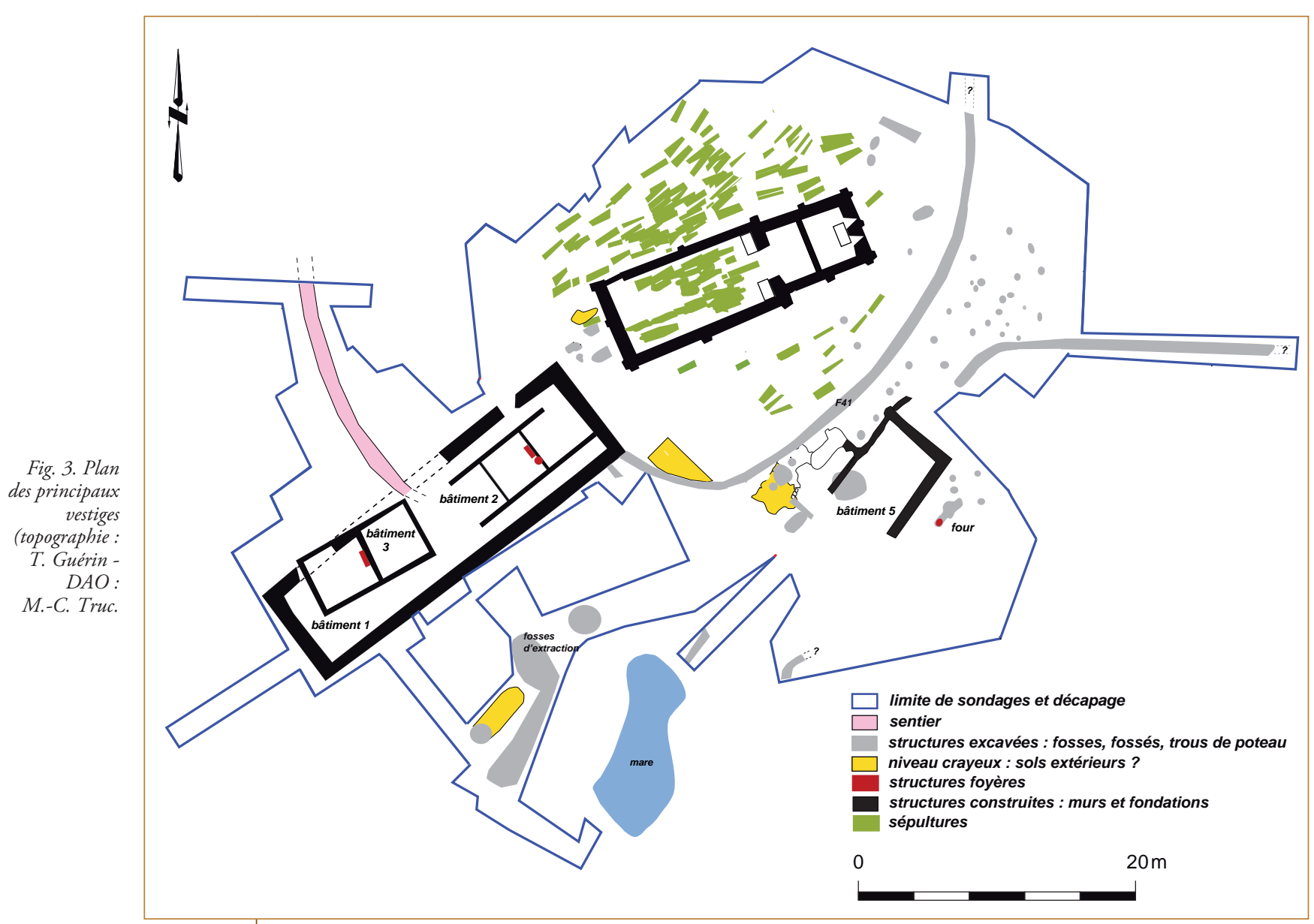

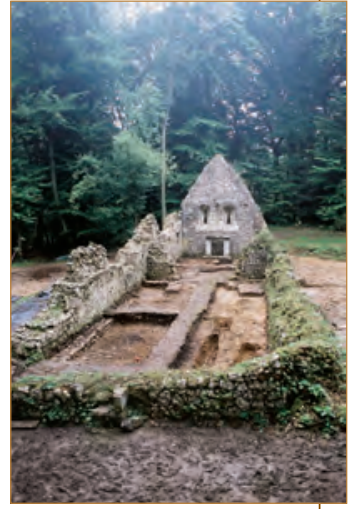

Fig. 4. Vue de la chapelle prise de l'ouest (cliché: M.-C. Truc).

Fig. 5. Stratigraphie du chour de la chapelle. Vue prise de l'ouest (cliché : M.-C. Truc).

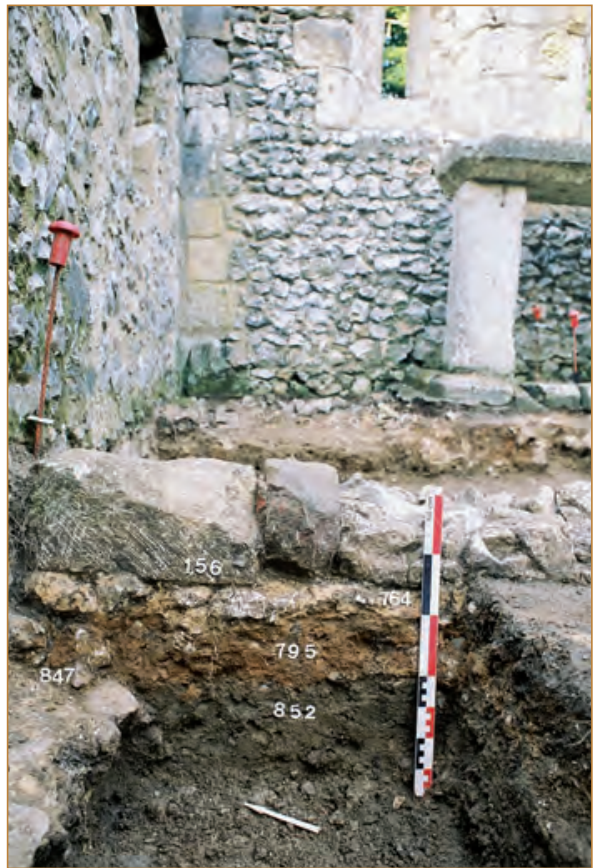

et mortier large de $1 \mathrm{~m}$ à $1,20 \mathrm{~m}$. Les angles, les ouvertures et les contreforts sont en gros appareil régulier de pierres calcaires taillées.

Peu après la construction des fondations de la chapelle, l'intérieur de l'édifice est nivelé au moyen d'un remblai, sans doute afin de compenser la déclivité naturelle du terrain du sud-est vers le nord-ouest. Ce remblai est surmonté d'un niveau de sol incendié $\mathrm{au} \mathrm{Xv}^{\mathrm{e}}$ siècle, retrouvé essentiellement dans le chœur et de façon plus ponctuelle dans la nef. Il est impossible d'affirmer que ce sol soit d'origine, un niveau de circulation plus ancien ayant très bien pu exister.

Après l'incendie, un emmarchement est construit dans le chœur afin de supporter l'autel (fig. 5). C'est sans doute vers cette période que sont installés les deux autels latéraux de la nef, dont ne subsistent plus que les fondations en craie et mortier.

Enfin, alors que le chœur n'a livré aucune sépulture, une trentaine de tombes ont été découvertes dans la nef. 


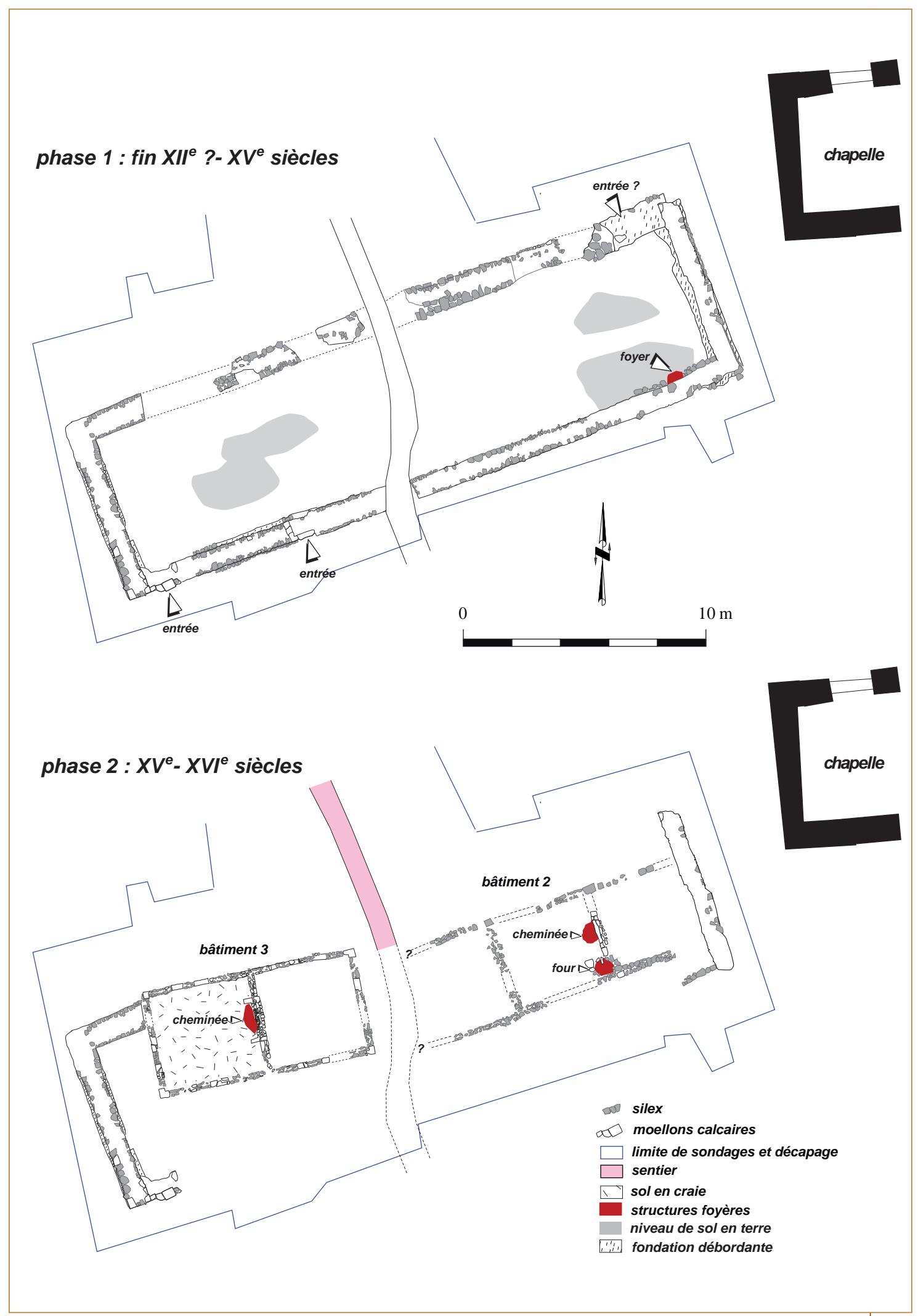

Fig. 6. La zone d'habitation (topographie: F. Le Roux - DAO : M.-C. Truc). 
Vers la même époque que l'édification de la chapelle, un vaste bâtiment en dur est construit à $3 \mathrm{~m}$ à l'ouest de celle-ci (fig. 3 et 6 : bâtiment 1 ). Long de $26 \mathrm{~m}$ et large de $7 \mathrm{~m}$, ses dimensions sont plus imposantes que celles de l'édifice cultuel. En revanche leurs matériaux et mode de construction sont en tous points identiques : parement de silex équarris, blocage de silex et mortier, emploi de la pierre calcaire taillée réservé aux angles et aux ouvertures.

Si deux entrées sont bien conservées dans le mur sud, une troisième, localisée dans le mur nord, est simplement suggérée par une pierre, retrouvée à proximité dans les déblais, qui pourrait être un linteau de porte. Ceci dit, la présence d'une ouverture à cet endroit semble logique, puisqu' elle donne ainsi sur la voie d'accès à la léproserie et ouvre du même côté que la chapelle.

L'intérieur du bâtiment 1 est mal conservé, en raison des réoccupations postérieures. Il ne subsiste notamment pas de trace de cloisonnement. Quelques lambeaux de sol en terre battue ont été repérés par endroit, ainsi qu'un petit foyer mural au sud.

La morphologie du bâtiment 1, la présence d'un foyer et l'absence de toute autre habitation sur le site à la même période, permettent de l'interpréter comme une habitation. La taille imposante

Fig. 7. Le dépôt monétaire (cliché : T. Cardon).

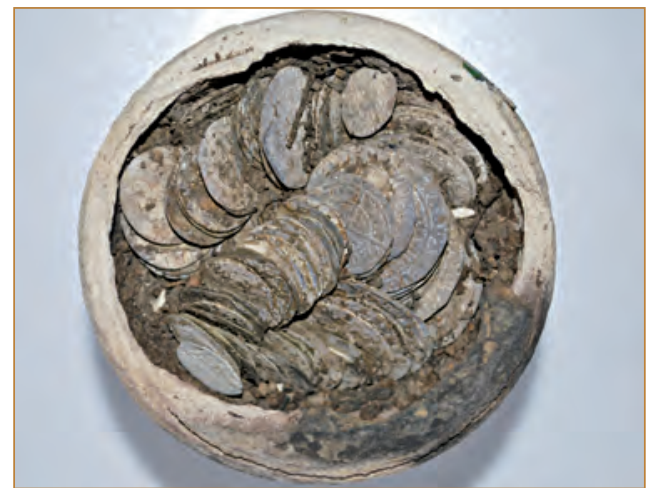

des murs suggère la présence d'un étage. Les nombreuses tuiles retrouvées dans les remblais indiquent une toiture couverte de ce type ce matériau. Ainsi restitué, ce bâtiment apparaît comme une construction imposante, semblable à ce que l'on trouve dans les prieurés de la même époque. Il est plausible que nous soyons en présence d'un habitat collectif, dans lequel devaient résider les malades et éventuellement le personnel soignant. Dans le courant du $\mathrm{Xv}^{\mathrm{e}}$ siècle, les murs gouttereaux du bâtiment 1 sont démontés, arasés et remblayés. Dans sa moitié orientale, reprenant la même orientation, est implantée une habitation plus petite (bâtiment 2) mesurant $8 \mathrm{~m} \mathrm{x} \mathrm{3,3} \mathrm{m} \mathrm{et} \mathrm{divi-}$ sée en trois pièces de taille égale, dont une n'était sans doute pas fermée (fig. 3 et 6 : bâtiment 2). Le pignon oriental est constitué par l'ancienne élévation du premier bâtiment qui est repris. Les murs gouttereaux sont des solins de silex non maçonnés, qui devaient isoler de l'humidité des élévations en matériaux périssables (murs en bois et torchis ?). Enfin le mur de refend séparant les pièces 1 et 2, qui reçoit l'appui d'une cheminée et d'un four accolés, est en moellons de craie. Les sols, non conservés, étaient sans doute en terre. Dans le sol extérieur a été aménagée une cache recelant un dépôt monétaire comprenant 290 monnaies d'argent anglaises des $\mathrm{XIV}^{\mathrm{e}}-\mathrm{XV}^{\mathrm{e}}$ siècles ainsi que 2 écus d'or français $d u x^{e}$. Les monnaies ont été triées puis soigneusement enserrées dans un tissu déposé dans une tirelire, elle-même contenue dans un pot à cuire calé par des briques. La somme est assez modeste, en accord avec les trouvailles isolées sur le reste du site. La composition et la disposition des monnaies laisse entendre une thésaurisation sur plusieurs années de petites sommes en bonne monnaie d'argent. La dernière manipulation de cette cache monétaire se situe vers 1465-1466. Les monnaies sont très probablement prélevées dans la circulation courante ${ }^{4}$ (fig. 7).

Peu après la construction du bâtiment 2, une troisième et dernière habitation est édifiée (fig. 3 et 6 : bâtiment 3). Son orientation ne reprend pas celle des précédentes, mais s'aligne sur celle de la chapelle. Cette maison est également implantée sur les ruines des murs gouttereaux du bâtiment 1 . Mesurant 8,45 m x 4,20 m, elle comporte deux pièces séparées par un refend en moellons de craie, auquel s'adosse une cheminée destinée à chauffer une pièce au sol en craie. Le bâtiment est assis sur des solins non fondés en moellons de silex et de craie, dont la disposition dessine un motif en damier, caractéristique de cette période en Normandie. Cette maison, utilisée durant le $\mathrm{xvI}^{\mathrm{e}}$ siècle, est le dernier indice archéologique d'une occupation stable sur le site.

Les bâtiments 2 et 3 n'ont pas été construits en 
même temps, mais ils ont pu être contemporains même si le bâtiment 3 a subsisté un peu plus longtemps. Il s'agit d'habitations destinées à une ou plusieurs personnes, avec une capacité d'accueil de toute façon plus restreinte que dans le bâtiment 1 . La présence concomitante de deux petites maisons laisse entrevoir un mode de vie plus individualisé que durant la phase précédente, peut-être lié à une baisse des effectifs, voire à la recherche d'un plus grand confort. Ces maisons semblent désertées vers la fin du Xvi ${ }^{\mathrm{e}}$ siècle.

Ces deux maisons sont desservies par un petit sentier venant du nord et constitué d'un cailloutis dense et compact, épais de $5 \mathrm{~cm}$ environ. Sa fouille a livré un denier tournois de la fin du $\mathrm{Xv}^{\mathrm{e}}$ siècle et une petite boucle en huit caractéristiques de la fin du XVI ${ }^{\mathrm{e}}$, voire du XVII ${ }^{\mathrm{e}}$ siècle.

Durant tout le Moyen Âge, l'espace situé au sud-est de la chapelle et des bâtiments semble dévolu aux activités domestiques de la vie quotidienne. Cet espace est séparé du cimetière par un fossé d'enclos, profond de $20 \mathrm{~cm}$ environ dans le substrat et large d'une trentaine de centimètres. D’après le mobilier retrouvé dans son remplissage, il semble comblé vers la fin du Moyen Âge (fig. 3 : F 41).

Ces activités sont suggérées par la présence d'un four domestique, de fosses d'extraction ayant servi ensuite de dépotoir, d'espaces de circulation en craie et d'une structure construite non maçonnée (fig. 3 : bâtiment 4), au plan ouvert, qui constitue peut-être les restes d'un enclos ou muret destiné à délimiter une zone précise (jardin, etc.).

Parmi les installations indispensables sur un lieu de vie figure le point d'eau. Une mare pavée de silex, fonctionnant par rétention des eaux de pluie, existe justement sur ce secteur. Sa date de création est inconnue, son curage ainsi que les recherches archéologiques sur ses abords n'ayant rien donné. Elle figure néanmoins sur un terrier de $1687^{5}$ et rien n'interdit de lui supposer une origine plus ancienne, liée au fonctionnement de la léproserie. En effet, au Moyen Âge, les lépreux n’ont en principe pas accès aux points d'eau utilisés par les biens portants. En l'absence de source et de puits sur le site, l'aménagement d'une mare semble logique. L'approvisionnement a pu également se faire à la Seine, qui coule à $1 \mathrm{~km}$ en contrebas, comme le suggère un sentier, figuré sur le terrier de 1687 sous le nom de "Sente aux malades". Ce dernier relie les berges du fleuve à la léproserie sans passer par le village d'Aizier et est toujours partiellement visible (fig. 1).

L'établissement est enserré par un talus et un fossé externe (fig. 8). Les fouilles ont montré que le talus n'était surmonté ni de palissade ni de mur. Un relevé topographique du houx et du fragon épineux (Ruscus aculeatus) appelé aussi petit-houx, mené par Thomas Guérin ${ }^{6}$ permet de proposer l'hypothèse d'une haie de houx et fragon. En effet, le relevé de ces deux espèces montre que leur implantation suit de très près les talus qui structurent le site, dont celui de la léproserie qui nous intéresse ici. Or le fragon n'est pas une plante présente dans la nature de façon spontanée. Sans intervention humaine, il ne s'éloigne guère du lieu où il a été initialement planté. Faute d'entretien, il a par ailleurs tendance à disparaitre spontanément (Guérin, 2005, p. 54-60). Ces observations nuancent les habitudes historiographiques nous représentant les léproseries séparées encloses de hauts murs afin de limiter les risques de contamination. Ce type d'aménagement, qui a pu exister pour des léproseries importantes ou périurbaines, a peu de raisons d'être pour un petit établissement isolé comme celui d'Aizier. En effet, la délimitation de l'enclos, sous forme de haie d'épineux, chose d'ailleurs connue pour le Moyen Âge en Normandie $^{7}$, a juste vocation de délimitation.

Le site est traversé par une cavée à peu près parallèle à la route actuelle et qui, à quelques dizaines de mètres à l'est du site, paraît la rejoindre (fig. 1 et 8 ). D'après la tradition locale, toutes deux reprennent le tracé d'une voie romaine indiquée sur la carte de Peutinger et l'itinéraire d'Antonin (Duval, 1980). Sur la carte $\mathrm{IGN}^{8}$, elle apparaît sous le toponyme de "Chemin Perrey " (fig. 1).

5 ADSM, terrier 296.

6 Travail réalisé par Thomas Guérin dans le cadre du PCR «Fortifications de terre en Haute-Normandie ", coordonné par A.-M. Flambard-Héricher (université de Rouen). Aizier a servi de cas d'étude à une partie de l'équipe du PCR puisque les hypothèses émises d'après les résultats des relevés microtopographique et floristiques, pouvaient être vérifiés par l'ouverture de sondages, ce qui nétait pas toujours les cas des sites catraux prospectés. Son étude est notamment consignée dans le rapport de fouille 2005 (Guérin, 2005 , p. 54-60).

7 Maneuvrier, 2000.

8 IGN 1/25000, 1811 est. 
Cet voie reliait Lisieux (rive gauche de la Seine) à Lillebonne (rive droite) en passant par PontAudemer et traversait la Seine à Vieux-Port. Un embranchement permettait cependant de rejoindre Aizier en passant par la chapelle SaintThomas pour continuer vers Vatteville-la-Rue en longeant la Seine (Legros, 1984, p. 2 ; Lequoy, 1995 , p. 115 ; Duval, 1980 et 1986). Des sondages pratiqués sur son tracé à Aizier en 1987 , avaient révélé des phases successives d'utilisation, qu'il n'avait malheureusement pas été possible de dater ${ }^{9}$.

Dans la partie ouest de notre site, la voie est matérialisée par une cavée à fond plat, large de 3 à $4 \mathrm{~m}$ et dont la profondeur diminue d'ouest en est $(1,50$ à $1,20 \mathrm{~m})$. Dans la moitié orientale du site, elle n'est plus visible et son tracé n'a été repéré que grâce à la microtopographie et aux fouilles. Les fouilles ont révélé un chemin large de 1 à $3 \mathrm{~m}$, matérialisé par un cailloutis dense et compact, épais de 5 à $10 \mathrm{~cm}$. Aucune recharge, aucune ornière, aucun fossé bordier ni aucun mobilier datant n'ont été mis en évidence.

En l'absence d'élément de datation, nous n'avons pu ni infirmer ni confirmer une possible origine antique. En revanche, une utilisation de cet axe à l'époque médiévale ainsi qu'une origine plus ancienne que celle de la léproserie, nous semblent fort probables. En effet, l'organisation de l'établissement hospitalier semble respecter le tracé de la voie : la chapelle et les bâtiments en reprennent l'orientation et c'est d'ailleurs peut-être la raison pour laquelle la chapelle n'est pas rigoureusement orientée est-ouest. En outre, l'entrée de la chapelle se fait côté voie. De plus, le cimetière s'insère parfaitement entre le mur nord de la chapelle et la voie, aucune tombe ne venant la recouper (fig. 8). Enfin le terrier de 1687 (certes postérieur à la léproserie) montre que le site est traversé par une route reliant Aizier à Pont-Audemer.

Ce schéma correspond à ce qui a souvent été mis en évidence par les historiens, à savoir l'implantation systématique des léproseries le long d'un passage, ce qui permet non seulement le ravitaillement de l'établissement, mais également l'hébergement des voyageurs, notamment des lépreux errants. En outre, divers témoignages écrits nous apprennent que les lépreux vivant dans les léproseries avaient l'habitude de se poster à l'entrée, sur la route, afin de mendier (Uhrmacher, 2000, p. 21).

\section{Le prieuré \\ (milieu du XVI $I^{e}$ fin du XVIII ${ }^{e}$ siècle)}

Les fouilles ont montré un abandon des espaces de vie après le $\mathrm{XVI}^{\mathrm{e}}$ siècle : en effet les seules traces de fréquentation postérieure n'ont été repérées que dans la chapelle et sur ses abords immédiats.

Si lors de sa désaffection, le bâtiment 1 avait fait l'objet d'un nivellement et d'une récupération des matériaux, en revanche les deux autres bâtiments sont abandonnés en l'état au cours du $\mathrm{XvI}^{\mathrm{e}}$ siècle. Le bâtiment 3 s'écroule sur lui-même et le mur de refend intérieur est d'ailleurs retrouvé effondré sur presque toute sa hauteur. La cheminée et les solins, très bien conservés, ont échappé aux récupérateurs de pierre. Ces vestiges, juste nappés par une fine couche d'humus ne dépassant pas $10 \mathrm{~cm}$ d'épaisseur, restent détectables durant quatre siècles sous forme d'un monticule. Tous ces éléments semblent indiquer une absence d'occupation régulière du site. Toutefois, l'absence de récupération d'éléments de construction suggère que le lieu n'est pas non plus totalement abandonné et qu'il fait l'objet d'une fréquentation. L'activité se concentre désormais aux abords de la chapelle, comme le prouve la présence de quelques monnaies et de céramique moderne. Cette désaffection est confortée par les textes qui nous apprennent que la chapelle perd son rôle de maladrerie dans le courant $\mathrm{du} \mathrm{xvI}^{\mathrm{e}}$ siècle, pour devenir un prieuré simple à la nomination de l'abbé de Fécamp, c'està-dire sans charge d'âme ${ }^{10}$. Ce changement d'affectation intervient à une époque où une baisse de la fréquentation des léproseries semble apparaître dans les sources écrites (Bériac, 1988, p. 265-269). Dès lors, les prieurs titulaires de la chapelle et de ses maigres prébendes se succèdent. $\mathrm{Ne}$ résidant pas sur place, ils se contentent de percevoir les modestes bénéfices provenant de la vente de bois et de la location de quelques terres. Le lieu reste entretenu, comme le prouvent des mentions de réparations sur la chapelle au début du XvII ${ }^{\mathrm{e}}$ siècle : en 1614, le chancel est réparé et couvert de tuiles. Trois ans plus tard, un nouvel autel est mis en place. En 1627, des marchés sont passés pour réparer les maçonneries et la charpente de la nef ${ }^{11}$.

9 Sondages menés par Jean-Paul Ybert (Groupe archéologique, 1988).

10 ADSM, 7 H977 (cité par Yvernault, 2005, p. 20).

11 ADSM, $7 \mathrm{H} 977$ (ibid., p. 24-25). 


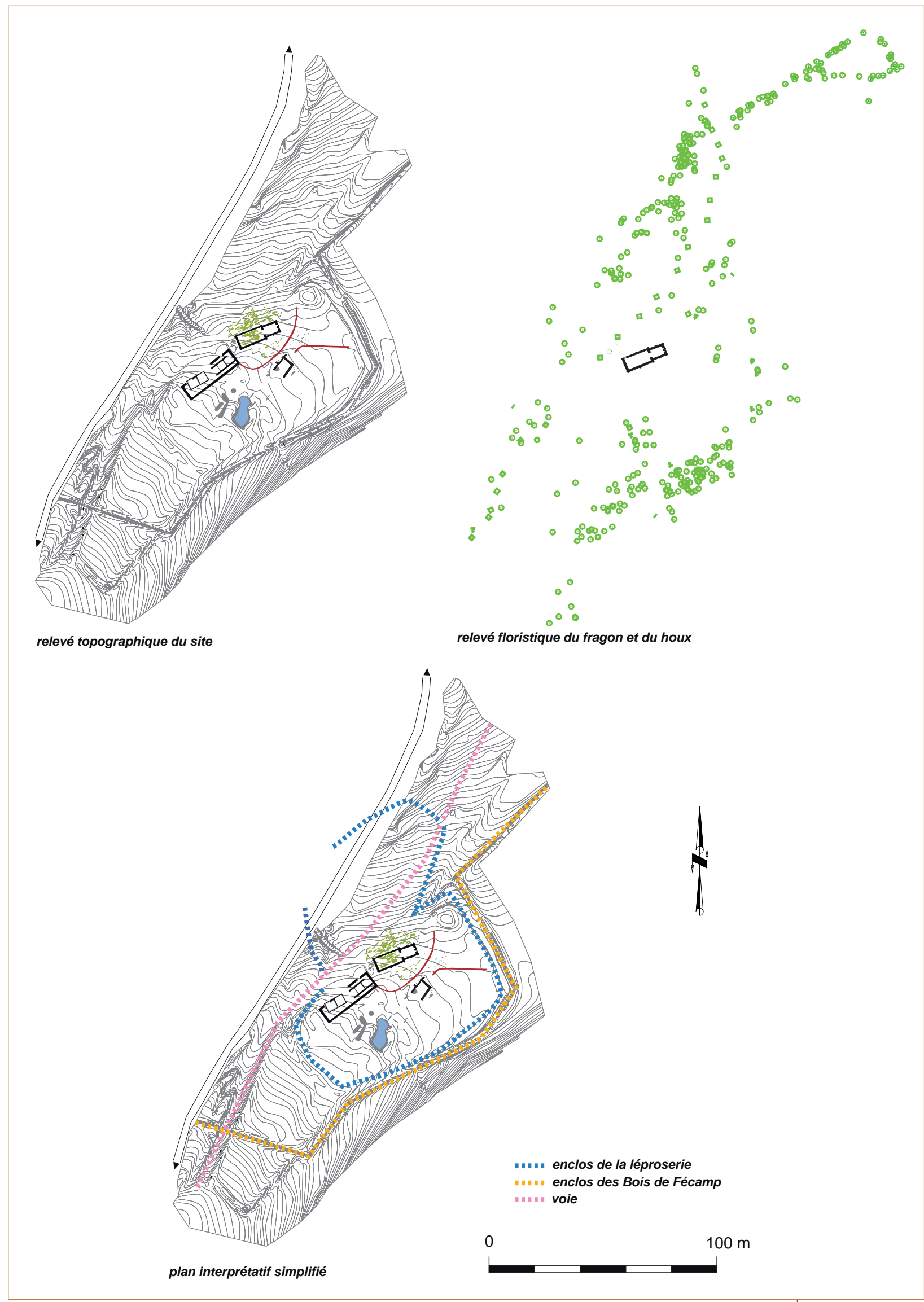

Fig. 8.

Les enclos et la voie (topographie: T. Guérin $D A O$ : M.-C. Truc). 
En 1641, des bénédictins réformés relevant de la Congrégation de Saint-Maur sont introduits dans le monastère de Fécamp, ce qui conduit à un partage des biens de l'abbaye. À partir de cette époque, les réparations sur la chapelle sont moins fréquentes et plus modestes, bien que quelques messes y soient encore célébrées de temps à autre, notamment le jour de la saint Thomas ${ }^{12}$. Toutefois les sources mentionnent sa dégradation avancée, à tel point qu'en 1717, suite à une visite de ClaudeMaur d'Aubigné, évêque de Rouen, la chapelle est frappée d'interdit ${ }^{13}$. À la fin du XvirI ${ }^{\mathrm{e}}$ siècle, toutes les visites indiquent qu'elle est en ruine. En 1770 , la chapelle est déclarée hors d'état de célébrer la messe, le toit est détruit, les murailles menacent de s'effondrer et la charpente est pourrie ${ }^{14}$. Lors de la vente des biens nationaux, la chapelle SaintThomas est probablement trop délabrée pour être vendue $^{15}$. Seules des pièces de terres dépendantes de la chapelle sont mises en vente.

Sans doute vers la fin du Moyen Âge, les Bois de Fécamp, c'est-à-dire la partie de l'actuelle forêt de Brotonne possédée par l'abbaye de Fécamp sur Aizier et ses environs - et dans laquelle la léproserie forme une enclave - sont entourés d'un enclos qui longe celui de la léproserie (fig. 8). La date précise de cet événement reste inconnue, mais d'après les sources, elle serait antérieure au milieu du $\mathrm{XVII}^{\mathrm{e}}$ siècle, car l'enclos est mentionné dès 1678 : «... Lesdits bois [de Fécamp] estoit fermé autrefoy par des fossés qui paroissent encior » et figure sur le terrier de 1687 (ADSM, 7H934, liasse 34). Peut-être même remonte-il à la fin du Moyen Âge, époque à laquelle un bornage est réalisé, afin de faire cesser les conflits de propriété concernant les bois de Fécamp à Aizier et Sainte-Croix-sur-Aizier (ADSM, 7H953, 13 e manuscrit). Cet enclos est constitué d'un fossé externe et d'un talus interne, tous deux bien conservés dans le paysage. Le talus, comme celui de la léproserie, était sans doute surmonté d'une haie composée de fragon et de houx, ainsi que semble le montrer l'enquête botanique (Guérin, 2005, p. 54-60). Cet enclos recoupe de façon très nette la voie dite antique, qui s'est décalée de quelques mètres vers le nord, à l'emplacement de la route actuelle dont nous ignorons malheureusement la date de percement.

\section{Le monde des morts}

\section{Les zones sépulcrales}

À l'issue de la campagne 2010, environ 250 individus ont été identifiés sur le site d'Aizier et un peu plus d'une trentaine de sépultures ont pu être complètement dégagées lors de l'ultime campagne (fig. 9).

Plusieurs zones sépulcrales ont été identifiées sur ce site : la plus importante se situe au nord de la chapelle avec de nombreuses tombes disposées suivant des alignements précis. Un deuxième secteur se trouve au sud de la chapelle, mais ne paraît que peu densément occupé. Les liens chronologiques entre les deux secteurs n'ont pu être déterminés, aucun mobilier n'ayant été trouvé avec les individus. Des datations ${ }^{14} \mathrm{C}$ sont prévues sur certains squelettes de ce secteur. Il est possible que cette partie du site ait été utilisée à partir de la saturation des espaces dévolus aux sépultures au nord de la chapelle. Le troisième secteur funéraire se trouve à l'intérieur même de la chapelle, où une trentaine de tombes ont pu être identifiées, uniquement dans la nef.

Avec l'importance grandissante des stages pratiques en archéologie dans le cadre des cursus universitaires ou professionnels, une attention toute particulière a été portée à la pédagogie et à la présentation des méthodes et des techniques de fouilles fines d'une sépulture sur le terrain. Une initiation aux méthodes d'enregistrement de l'anthropologie de terrain a été réalisée auprès des novices afin de leur faire découvrir toutes les étapes du dégagement d'une sépulture. Dans la plupart des cas, les fouilleurs ont suivi toutes les étapes de la fouille d'une sépulture (fouille, enregistrement, relevés altimétriques, photographie, démontage, conditionnement du matériel osseux). Les délais impartis à la fouille de plusieurs des tombes exhumées en 2010 dans les secteurs nord et nord-oriental du cimetière notamment ont été particulièrement restreints, comparables, voire plus courts, à ceux généralement impartis en contexte de sauvetage. Dans tous les cas cependant, les sépultures ont fait l'objet d'une étude de terrain complète. La méthodologie appliquée pour les sépultures, définie et

12 ADSM, 7H979 (ibid., p. 25).

13 ADSM, 7H977 (cité par Penna, 1999, p. 30).

14 Archives départementales de l'Eure (ADE), IIIF1 (cité par Yvernault, 2005, p. 29).

15 ADE, Q37 (cité par Yvernault, 2005, p. 29). 


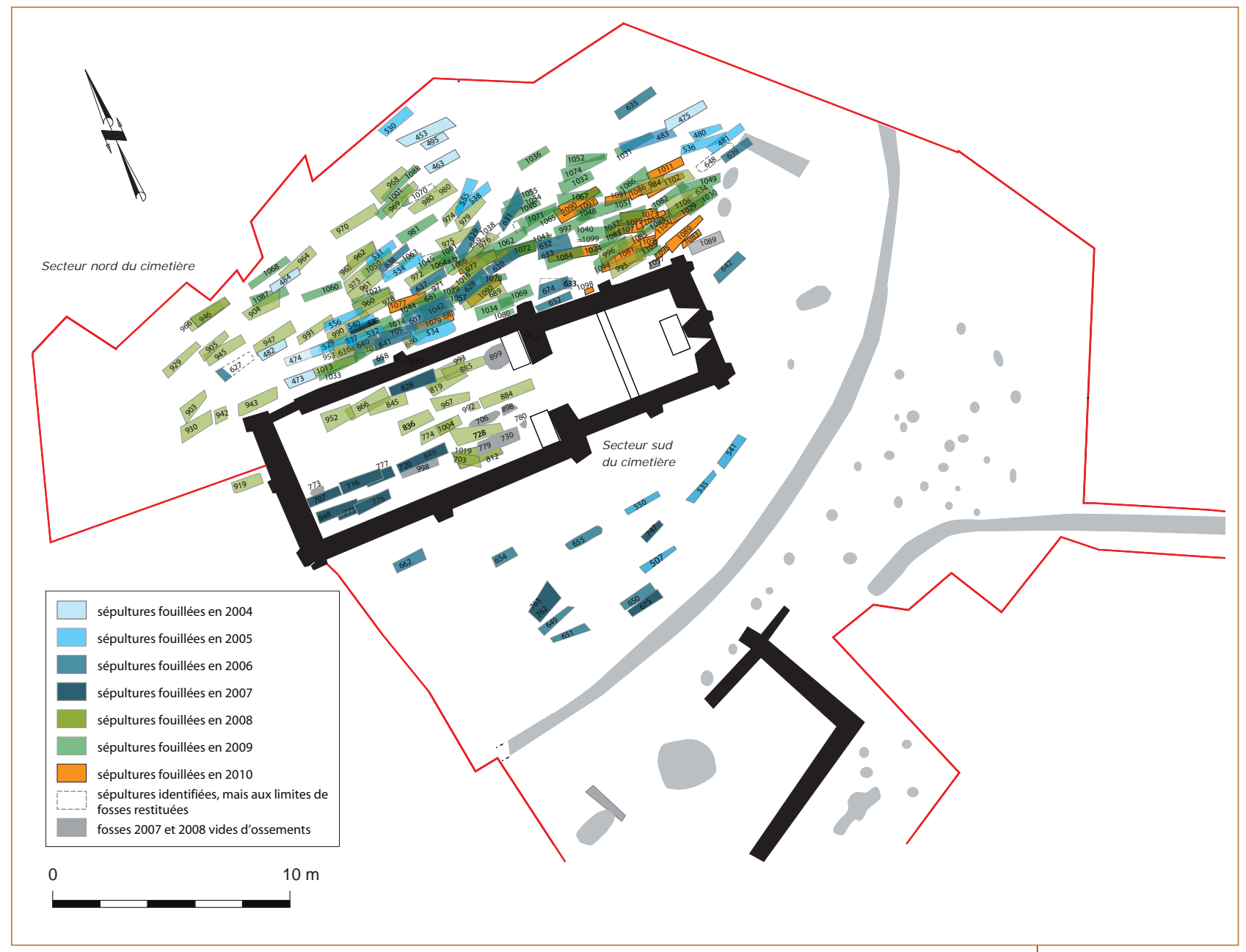

adaptée par Cécile Niel en fonction des urgences de la fouille et selon les priorités déterminées en début de campagne avec Marie-Cécile Truc, utilise les techniques généralement employées par l'anthropologie de terrain (fig. 10).

Parmi les 230 structures funéraires enregistrées sur le site d'Aizier, la grande majorité des inhumations est effectuée dans un coffrage de bois aménagé (58\%) (fig. 11) à l'aide de planches plus ou moins jointives, calées dans la plupart des cas par des silex placés sur les côtés ou sur le couvercle. 33 tombes (15\%) comportent un cercueil attesté par la présence de clous ou par des traces de bois retrouvées en négatif, 5 seulement sont en " terre libre " ou sans aménagement perceptible, mais elles comportent alors un linceul (2,6\%). 17 réductions d'un ou plusieurs individus ont également été individualisées sur le terrain (8\%), mais leur nombre est pour l'instant sous-estimé, la plupart des sépultures fouillées en 2009 et 2010 comportant des os placés en réduction et associés à la sépulture en place.

La taille des coffrages de bois varie de $0,51 \mathrm{~m}$ (pour un périnatal) à 2,10 $\mathrm{m}$ de long pour une largeur occidentale de 0,13 à $0,54 \mathrm{~m}$ et une largeur orientale de 0,09 à $0,49 \mathrm{~m}$. Les cercueils, moins nombreux, sont présents essentiellement dans les niveaux d'inhumation les plus récents. Ils se répartissent tout au long de la chapelle et sont avérés par la présence de très nombreux clous retrouvés tout autour des corps et à divers niveaux d'altitudes. Ainsi, dans plusieurs cas, les sépultures comptaient-elles de 10 à 31 clous. Les cercueils sont globalement de forme trapézoïdale et de dimensions moyennes : entre 0,67 m (sépulture immature) et 2,10 $\mathrm{m}$ de long pour une largeur occidentale de 0,15 à $0,47 \mathrm{~m}$ et une largeur orientale de 0,09 à $0,46 \mathrm{~m}$.

Pour 29 cas particulièrement mal conservés ou très partiels, il a été difficile de d'identifier la nature exacte du contenant dans lequel les
Fig. 9. Sépultures fouillées de 2004 à 2010 (topographie: T. Guérin DAO : T. Guérin, G. Marie, C. Niel, M.-C. Truc). 
Fig. 10.

Documents

types

remplis sur

le terrain

pour chaque

individu

exhuméà

Aizier et

traitement

des données

graphiques

en post-

fouille

(dessin: $U$.

Le Moigne,

C. Niel-

$D A O$ :

C. Niel).
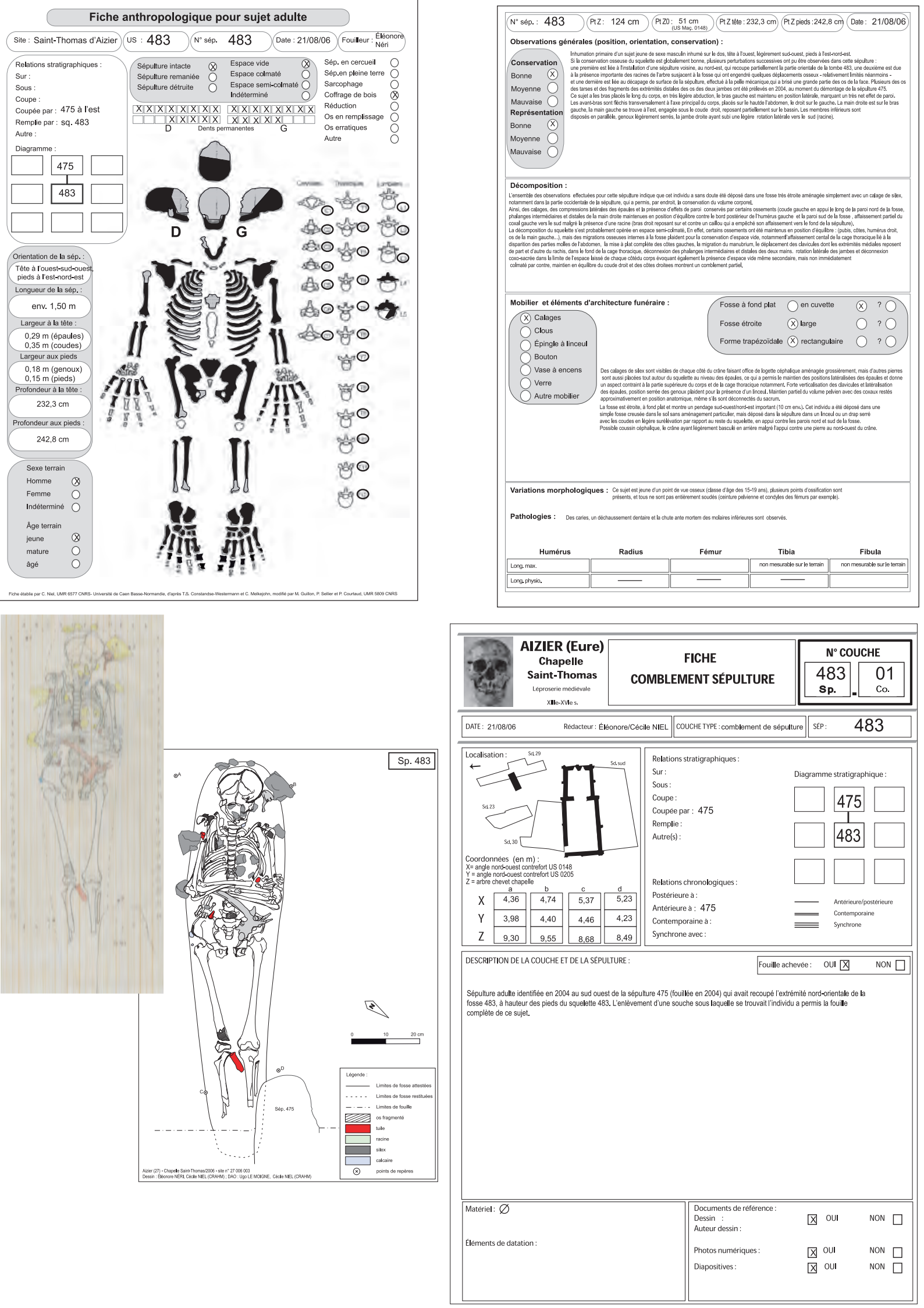


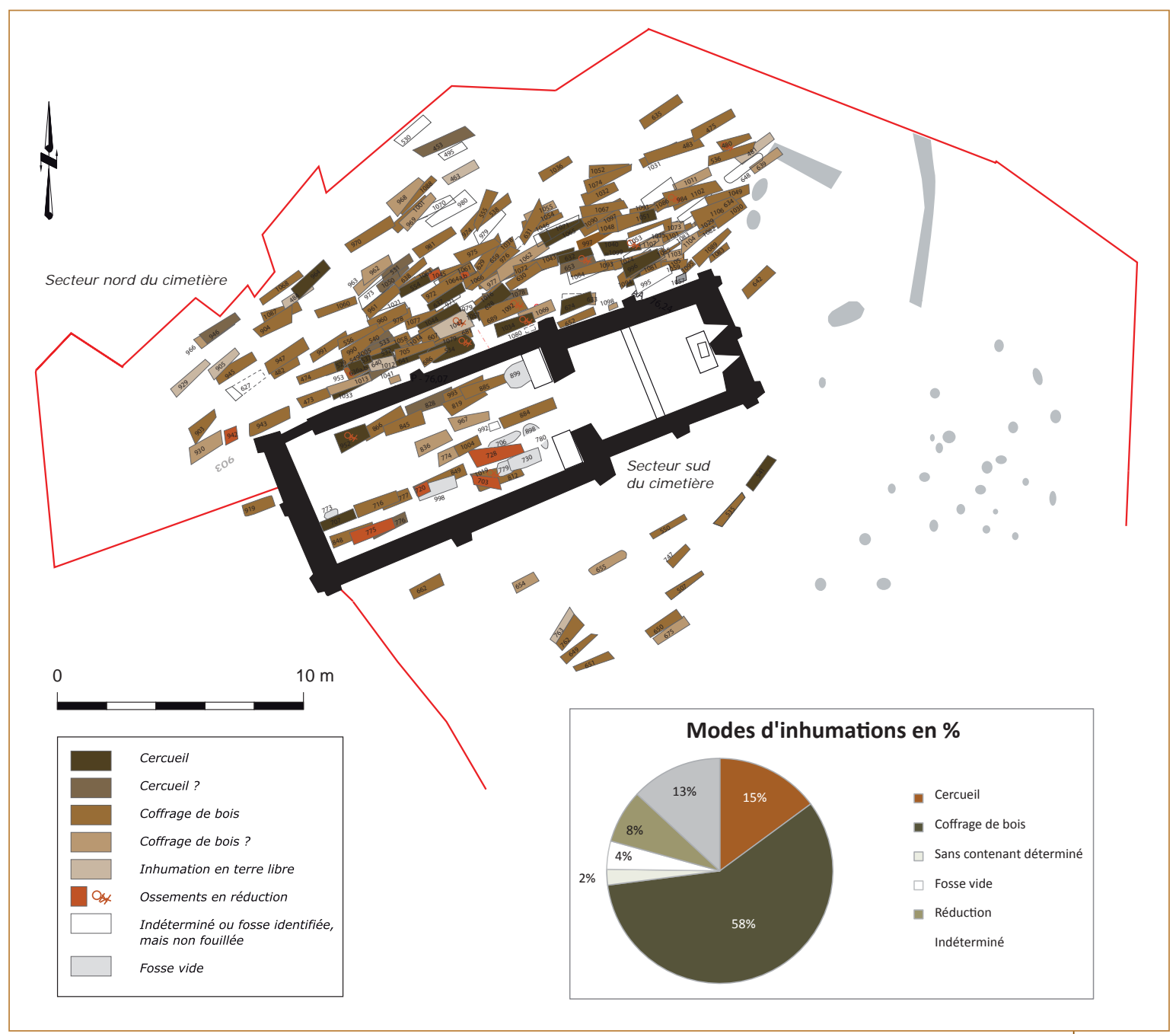

individus ont été inhumés. 129 coffrages de bois sont décomptés à Aizier. Ils sont attestés à la fois par des traces ligneuses brunes dans le sol ou par des effets de paroi en négatif très nets, les planches de bois qui servaient à protéger le cadavre ayant disparu, mais des espaces vides matérialisant encore leur présence et apparaissant très nettement au moment du décapage superficiel de la fosse. Des effets de parois sont aussi observés dans certains cas, alors même que la décomposition des corps s'est visiblement déroulée en espace vide, comme l'attestent certains déplacements osseux. Des calages céphaliques ou des pierres placées le long des parois, aux extrémités ou aux angles de certaines fosses ont aussi été notées dans plusieurs sépultures (Henrion, 2008).

Si, parmi toutes les sépultures exhumées à Aizier, la position de dépôt des corps est le plus fréquemment très simple et correspond aux positions généralement observées dans d'autres cimetières médiévaux (c'est-à-dire sur le dos avec les bras placés le long du corps, avant-bras fléchis sur l'abdomen, le thorax ou le pubis, les membres inférieurs étant disposés en parallèle dans l'axe du corps...), des positions singulières de dépôt ont plusieurs fois pu être observées (fig. 12). Pour plusieurs de ces cas, l'hypothèse de l'utilisation d'un coffre-meuble ou d'une huche à des fins funéraires peut être proposée, la taille de ces contenants particuliers (de $1,20 \mathrm{~m}$ à $1,40 \mathrm{~m}$ de long pour une largeur de 0,20 m à 0,35 m maximum) étant difficilement compatible avec celles des sépultures en cercueil ou en coffrage de bois identifiés par ailleurs à Aizier.

En confirmation des observations effectuées lors des années antérieures à 2010, la plus forte densité de sépultures se trouve dans la zone accolée à la chapelle avec des recoupements d'inhumations et un nombre de sujets réduits important. Jusqu'à six niveaux d'inhumations successifs ont d'ailleurs pu être reconnus dans ce secteur et les schémas stratigraphiques, en cours de réalisation, révèlent des relations plus complexes que ce qui avait pu être imaginé au départ et semblent eux aussi
Fig. 11. Répartition des modes d'inhumation (topographie: T. Guérin $D A O: T$. Guérin, G. Marie, C. Niel, M.-C. Truc). 

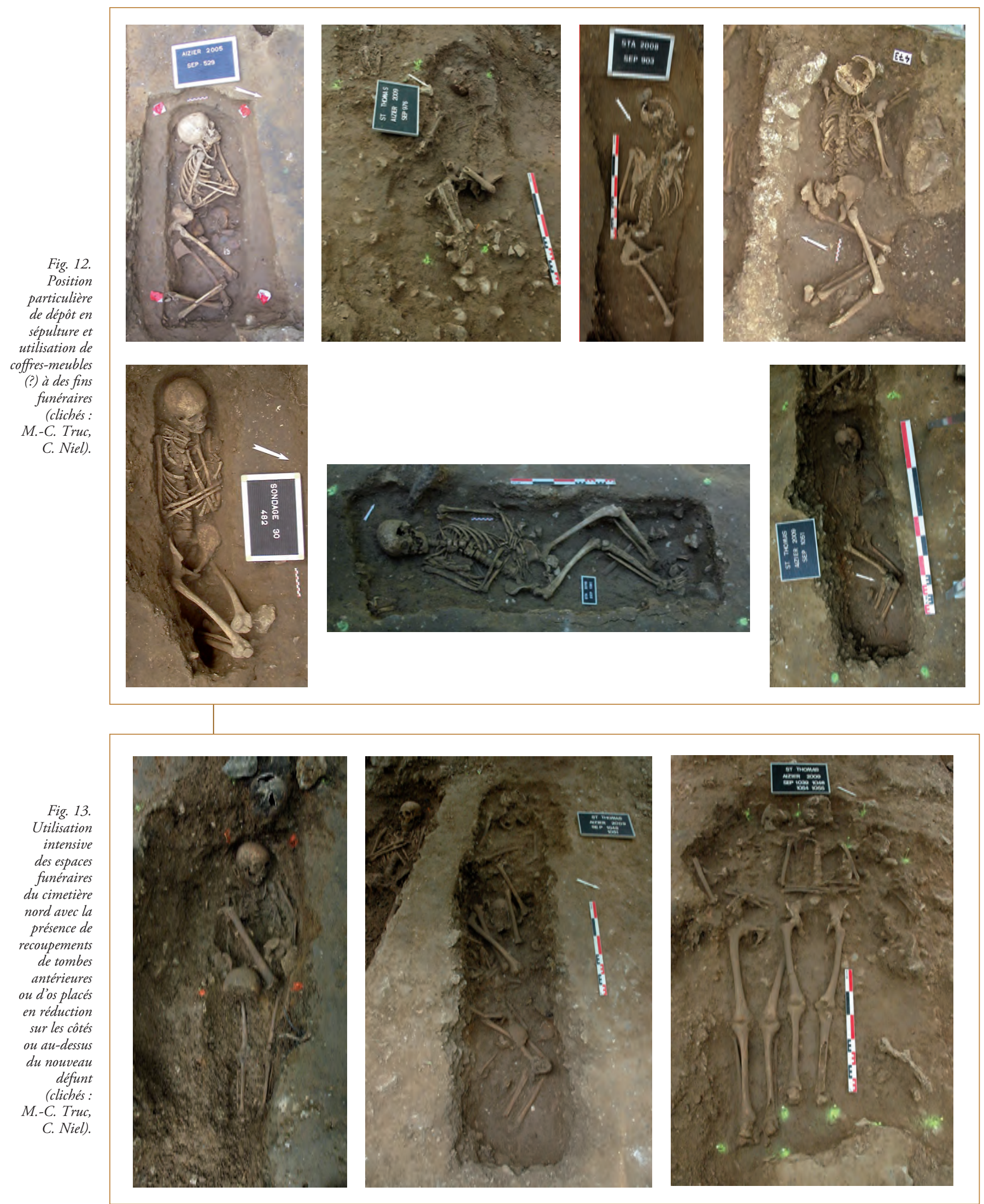
appuyer l'idée d'une gestion rigoureuse du cimetière nord. Plusieurs datations ${ }^{14} \mathrm{C}$ seront d'ailleurs à prévoir lors des travaux de post-fouille pour affiner le phasage des sépultures et permettre ainsi de caler chronologiquement les tombes les unes par rapport aux autres. Dans une grande partie de ces sépultures, les nombreux ossements placés en réduction sur les pourtours de la fosse, déposés au-dessus de l'inhumé en place ou retrouvés dans la terre de comblement attestent d'une gestion attentive et soigneuse des espaces funéraires par les fossoyeurs. Aucun ossuaire ou fosse commune n'a pour l'instant été identifié dans ce cimetière et à chaque fois qu'une inhumation perturbe une tombe antérieure, les os du ou des premiers occupants ont soigneusement été ramassés et redéposés dans la nouvelle fosse (fig. 13). Les analyses menées en laboratoire sur plusieurs des sujets perturbés par des inhumations postérieures nous permettent également d'appuyer cette hypothèse. Nous avons en effet pu compléter, de manière parfois importante, certains individus partiels à l'aide des ossements trouvés en réduction dans les tombes adjacentes ou postérieures qui avaient bouleversé leur sépulture. Ces observations semblent témoigner d'un certain respect de la mémoire des défunts et pourraient indiquer une connaissance pérenne des secteurs d'inhumation au sein du cimetière, peut-être en fonction de critères sociaux ou en liaison avec des groupes familiaux.

En effet, nous avons pu remarquer que dans un peu plus d'une vingtaine de cas, des sujets ont été inhumés dans une sépulture immédiatement sus-jacente ou recoupant presque exactement une tombe antérieure (fig. 14), et ce phénomène répétitif ne semble pas lié uniquement au hasard, mais permet d'avancer plusieurs hypothèses, dont la gestion très rigoureuse du site. Les tombes sont en effet placées selon des alignements relativement réguliers et, lorsque des recoupements de fosses sont observés, les os des défunts antérieurs sont visiblement soigneusement ramassés et placés en réduction. Il est aussi possible que la signalisation des tombes ait longtemps perduré et les inhumations localisées à l'endroit précis de sépultures antérieures pourraient souligner la volonté des défunts ou de leur famille d'être enterrés à un endroit en particulier, peut-être dans le cadre, là encore, d'un rapprochement familial.

Plusieurs caractères osseux discrets communs aux individus inhumés à proximité les uns des autres ont d'ailleurs pu être relevés tant sur le terrain qu'en laboratoire et appuient également cette première interprétation. Par ailleurs, le nombre de sépultures identifiées rapporté à la durée d'utilisation à des fins funéraires des divers espaces autour de la chapelle permettent de calculer qu'il n'y avait en réalité que peu de décès (à peine un décès tous les deux ans) et qu'il ne devait pas être difficile de conserver le souvenir de l'emplacement précis des tombes les unes par rapport aux autres.

\section{Bilan préliminaire de l'analyse paléoanthropologique}

L'analyse de laboratoire menée depuis 2004 a d'ores et déjà permis d'étudier 186 individus pour lesquels un dossier individuel réunissant les mesures ostéométriques et les observations morphologiques a été réalisé (selon un corpus de 600 mesures et observations utilisées au laboratoire de paléoanthropologie du CRAHAM).

La diagnose sexuelle, basée sur la morphologie et les dimensions du bassin, qui est considéré comme l'élément osseux le plus discriminant en anthropologie, a pu être réalisée pour 125 individus (dont 2 grands adolescents).

La répartition spatiale des sépultures masculines et féminines récapitule l'ensemble des informations actuellement disponibles pour l'ensemble des sépultures fouillées entre 2004 et 2010 (fig. 15), à partir de l'étude anthropologique en laboratoire et de la diagnose sexuelle préliminaire réalisée au moment de la fouille.

Elle confirme une répartition équilibrée des sexes sur l'ensemble de la population inhumée dans le cimetière. Si la distribution spatiale de ces tombes ne semble pas montrer de concentrations particulièrement significatives en faveur d'un sexe ou de l'autre dans les deux principaux secteurs d'inhumations, au nord et au sud de la chapelle, le nombre de sépultures féminines ou supposées féminines actuellement comptabilisé n'est pas supérieur dans la zone nord ( 43 femmes pour 42 hommes).

Toutefois, le nombre de sujets de sexe indéterminable (ossements trop médiocrement conservés ou trop partiels) ou encore non déterminé tout comme l'étude encore incomplète de cette partie de la population du cimetière interdisent, pour l'instant, d'en tirer des conclusions réellement significatives. À l'inverse, une nette prédominance masculine est observée pour les sujets inhumés à l'intérieur de la chapelle : seules quatre femmes pour douze hommes sont décomptées.

Cette prédominance dans le sanctuaire est courante et a déjà été observée sur plusieurs autres sites aux mêmes périodes comme dans le cimetière 
Fig. 14. Persistance

de la

signalisation

des tombes? (clichés :

M.-C. Truc, C. Niel).
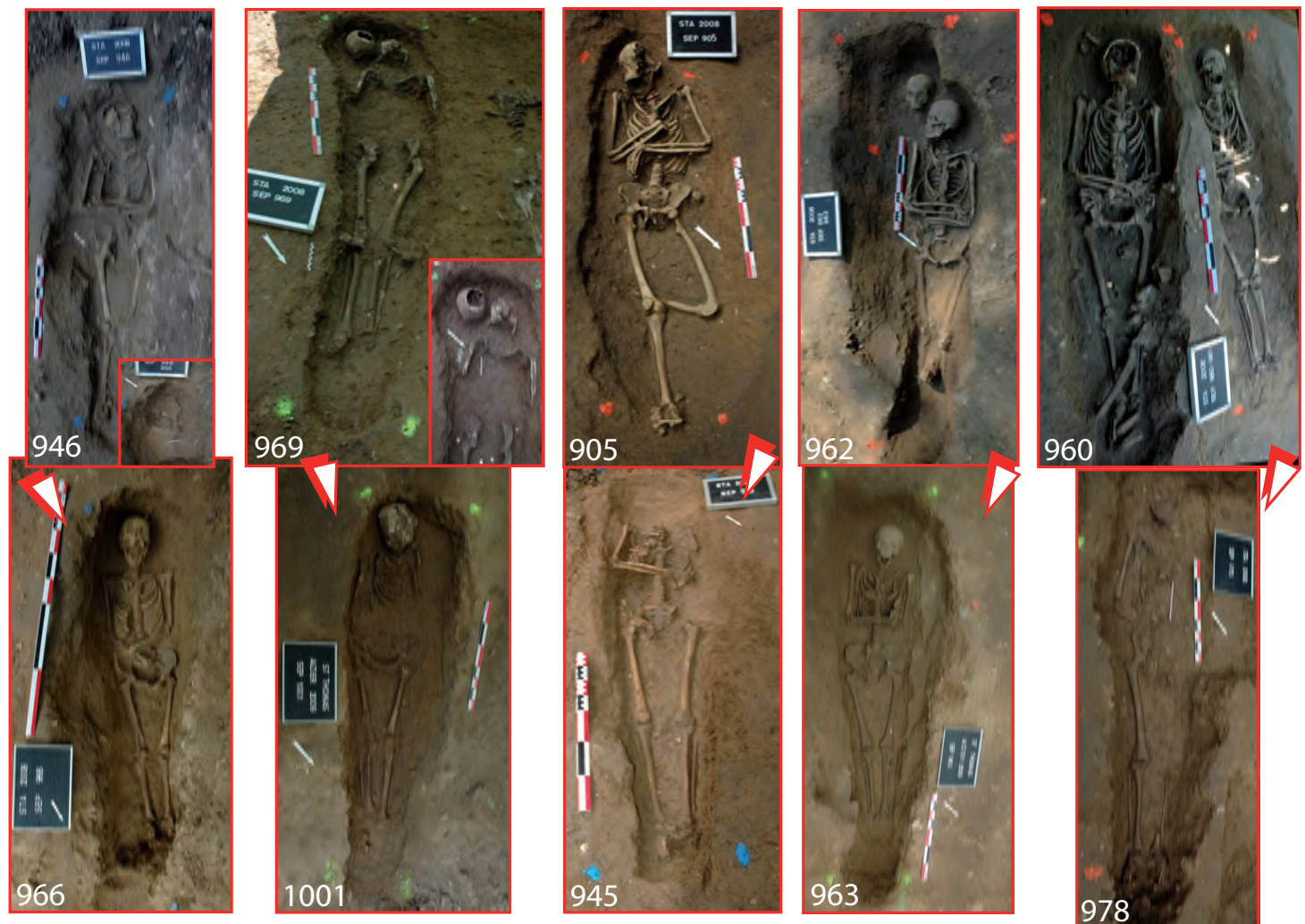

Dans une vingtaine de cas, des sujets ont été inhumés dans une sépulture immédiatement sus-jacente ou recoupant presque exactement une tombe antérieure. Ce phénomène répétitif ne semble pas simplement lié au hasard, mais suggère plusieurs hypothèses : gestion très rigoureuse du cimetière avec alignements de tombes réguliers, ramassage soigneux des os des défunts antérieurs systématiquement placés en réduction, signalisation des tombes dans la durée... Le fait d'installer des défunts à l'endroit précis d'une sépulture antérieure pourrait également souligner la volonté des défunts ou de leur famille d'être enterré à un endroit en particulier, peut-être dans le cadre d'un rapprochement familial.
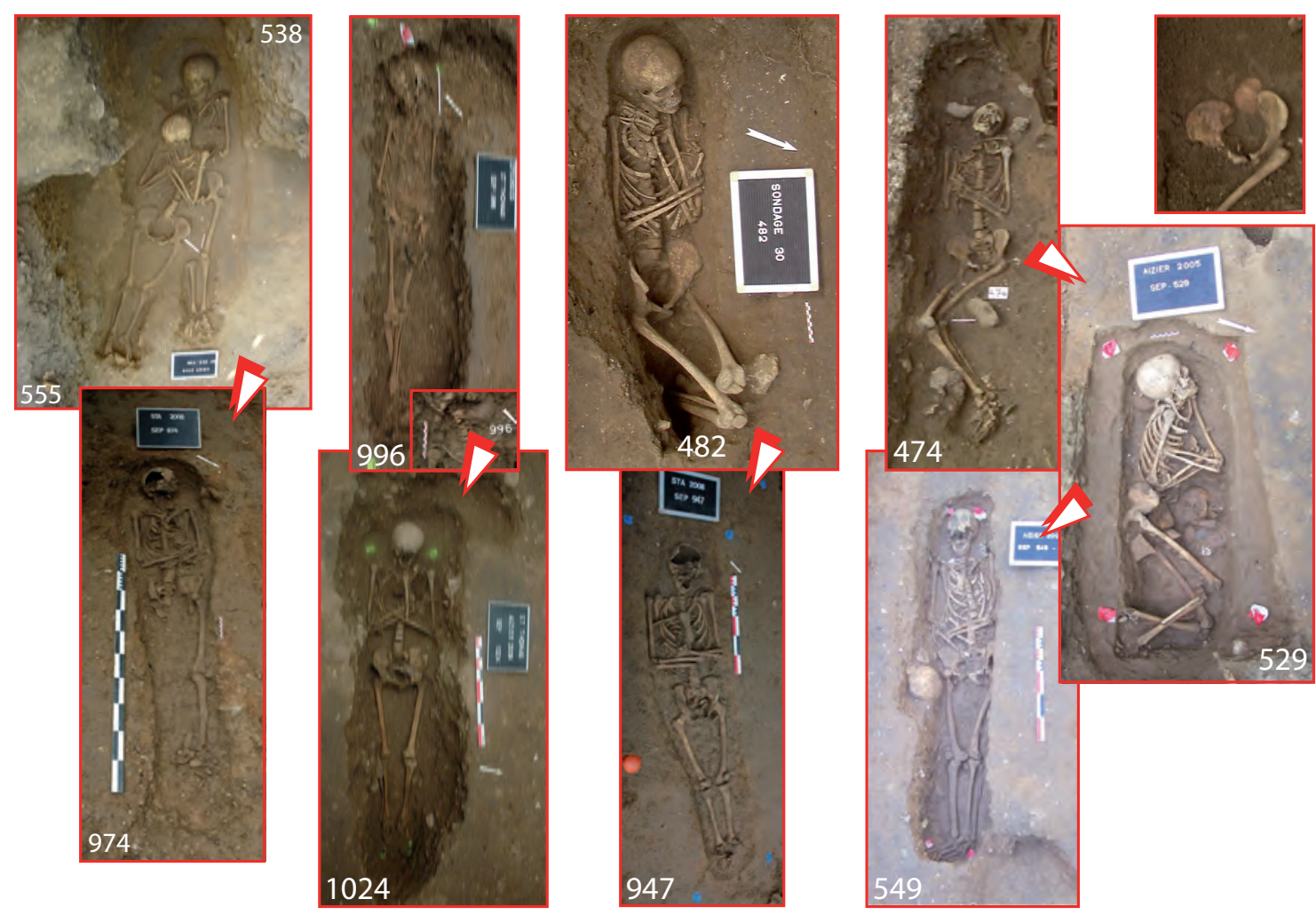


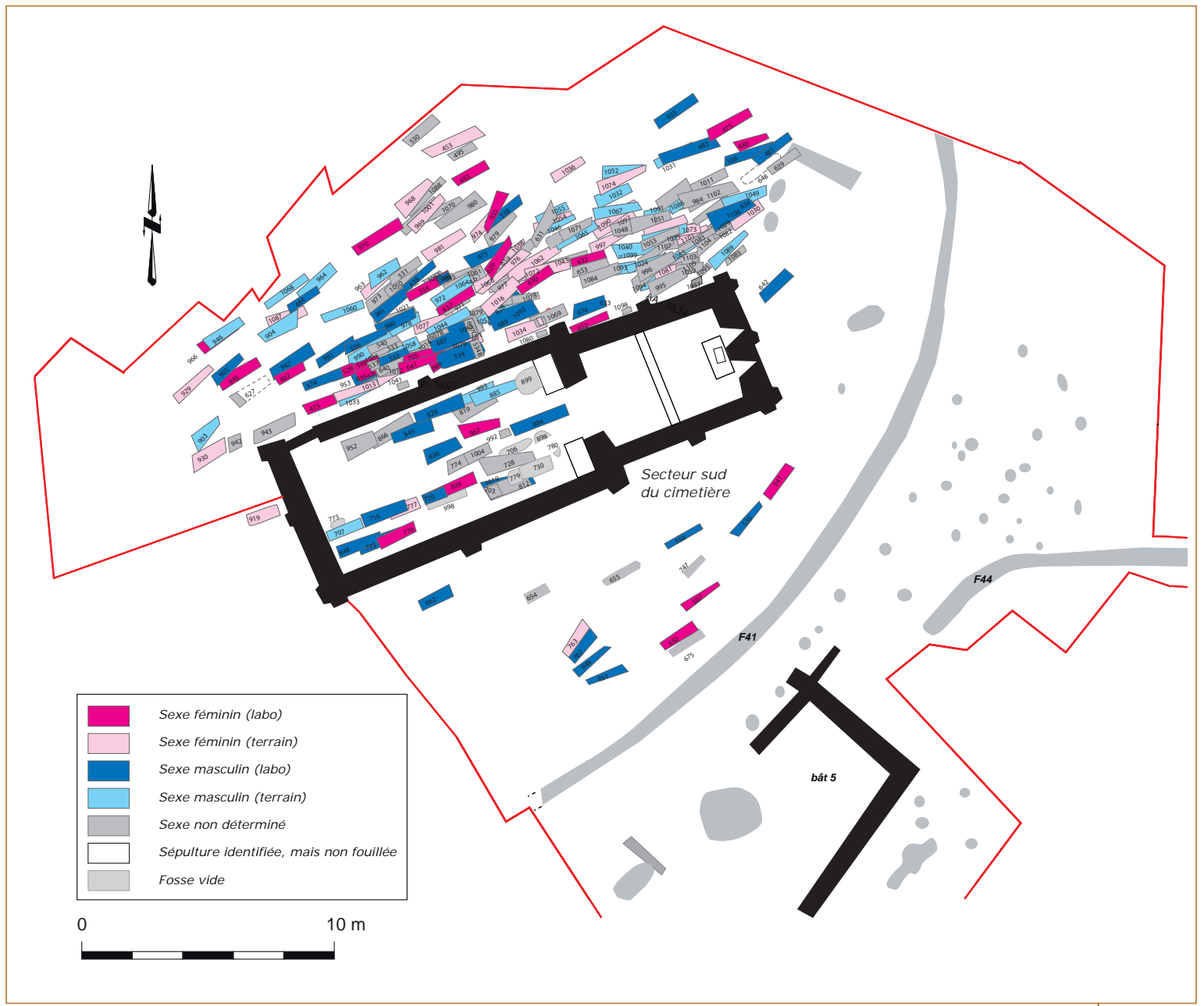

de la Cour d'Albane à Rouen ou dans le chœur de l'église Saint-Pierre de Thaon (Calvados). L'analyse complète réalisée sur les squelettes issus de la nef permet de préciser que cette dernière a pu constituer une zone d'inhumation privilégiée pour les hommes et que, parmi les inhumés (hommes et femmes), certains étaient des lépreux.

La majorité des malades recensés actuellement parmi les défunts d'Aizier (une cinquantaine) sont localisés dans le cimetière nord où la densité des inhumations est particulièrement importante avec des rapprochements ou des recoupements de fosses qui ne semblent pas toujours fortuits (fig. 16). Les autres secteurs funéraires ne sont pas pour autant exempts de malades : quelques cas avérés ou probables ont été décelés dans le cimetière sud (5 cas) et les indices flagrants d'une affection lépromateuse ont été observés sur le squelette d'un adolescent d'une quinzaine d'années inhumé au chevet de l'édifice.

Sans anticiper sur la suite de l'étude, il ressort clairement que bon nombre des individus les plus gravement touchés présentent les indices macroscopiques caractéristiques de la lèpre lépromateuse (fig. 17), confirmant ainsi une spécialisation de l'aire funéraire en rapport avec la vocation hospitalière de l'établissement, mais des ressemblances morphologiques ont également pu être constatées entre les occupants de tombes géographiquement très proches et suggèrent un recrutement plus complexe. D'autres squelettes présentent également des lésions évocatrices d'une maladie infectieuse, mais ces atteintes sont trop isolées ou insuffisamment discriminantes par rapport à d'autres étiologies possibles pour être retenues parmi les suspicions de lèpre.

Plusieurs cas de traumatismes divers des membres supérieurs, des mains et des membres inférieurs ont aussi été notés, de même que la présence d'atteintes dégénératives importantes, parfois en association avec la lèpre. Plusieurs individus fortement handicapés de leur vivant par des malformations congénitales sont également présents parmi les défunts. L'étude paléopathologique approfondie de tous ces individus est en cours au Centre d'études paléopathologiques du Nord, sous la responsabilité de Joël Blondiaux, qui essaiera d'établir si ces manifestations atypiques sont en
Fig. 15. Répartition sexuelle des individus (identification de terrain et de laboratoire). Topographie : T. Guérin -

DAO : T. Guérin,

G. Marie, C. Niel

M.-C. Truc. 


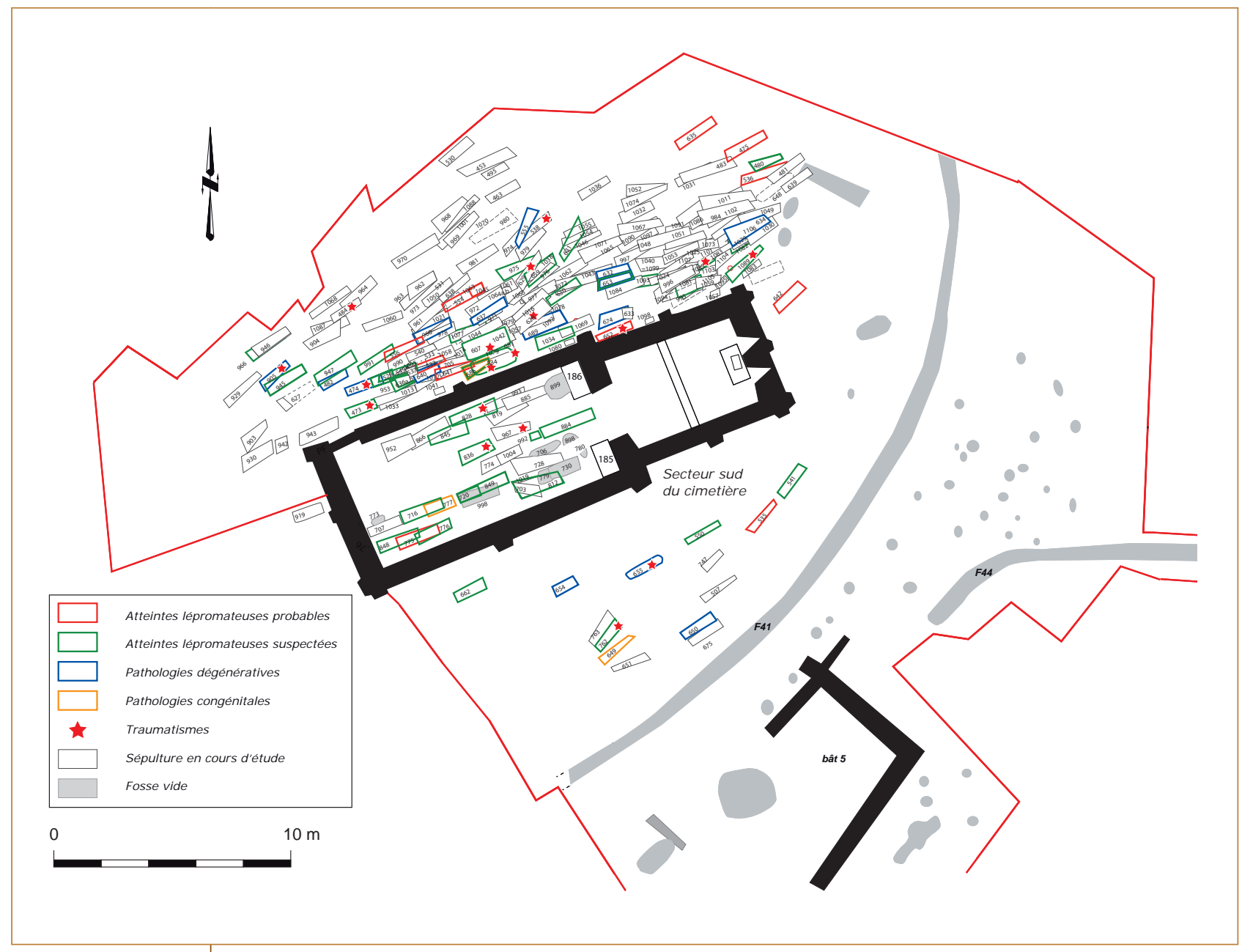

Fig. 16. Répartition des atteintes lépromateuses (d'après les observations de terrain et de laboratoire).

Topographie: T. Guérin DAO : T. Guérin, G. Marie, C. Niel, M.-C. Truc. rapport avec l'affection lépromateuse ou si d'autres maladies concurrentes, comme la tuberculose par exemple, sont aussi représentées.

La plupart des individus examinés en laboratoire, y compris ceux dont le squelette paraît indemne de toute autre atteinte, ont un état bucco-dentaire extrêmement médiocre, caractérisé par de volumineux dépôts tartriques, une résorption parodontale très accentuée, de multiples caries occasionnant parfois la destruction partielle ou totale des couronnes et associées le plus souvent à des lésions kystiques. La fréquence élevée des hypoplasies (anomalies de développement de l'émail lors de la formation dentaire), observées sur plus de la moitié des dentures adultes, confirme l'état sanitaire précaire de cette population hospitalière, certainement largement généré par de mauvaises conditions alimentaires durant la petite enfance. La fouille complète des dernières sépultures du cimetière extérieur est désormais achevée et leur étude en laboratoire permettra d'obtenir une vision de la population inhumée et de vérifier s'il existe des différences significatives dans la gestion et la destination des divers espaces sépulcraux. La richesse des informations obtenues à l'issue de ces premiers travaux et les larges perspectives ouvertes, tant pour l'identification des pathologies anciennes, leur taux de prévalence et leur développement que pour l'histoire des malades et de leur environnement social et économique, confirment le potentiel exceptionnel du site de la chapelle Saint-Thomas d'Aizier comparé aux rares cimetières de léproserie récemment abordés par l'archéologie.

\section{Conclusion}

Même si l'étude n'est pas encore achevée, les premières données permettent déjà de se faire une idée de cet établissement hospitalier. Il ne parait pas être 

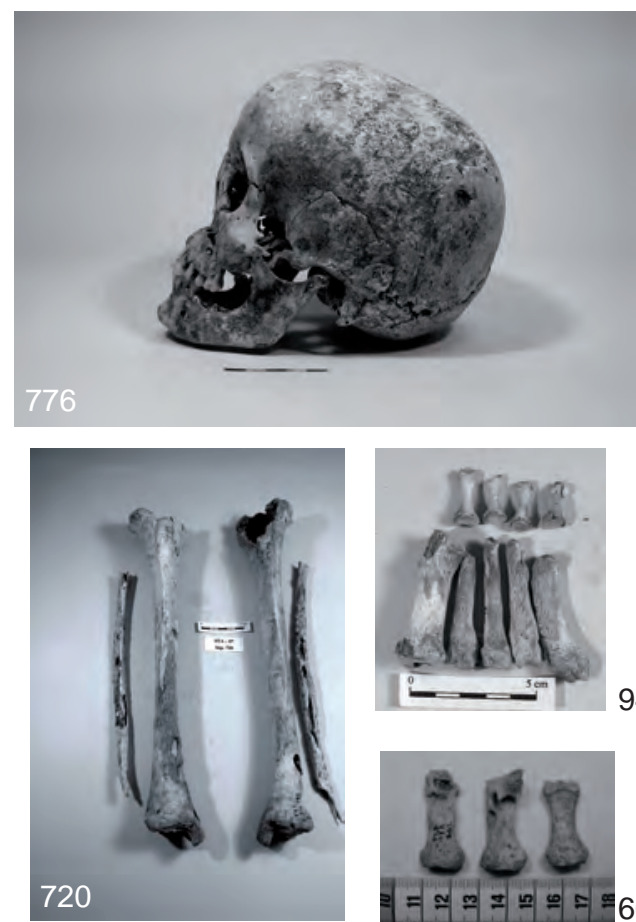

945
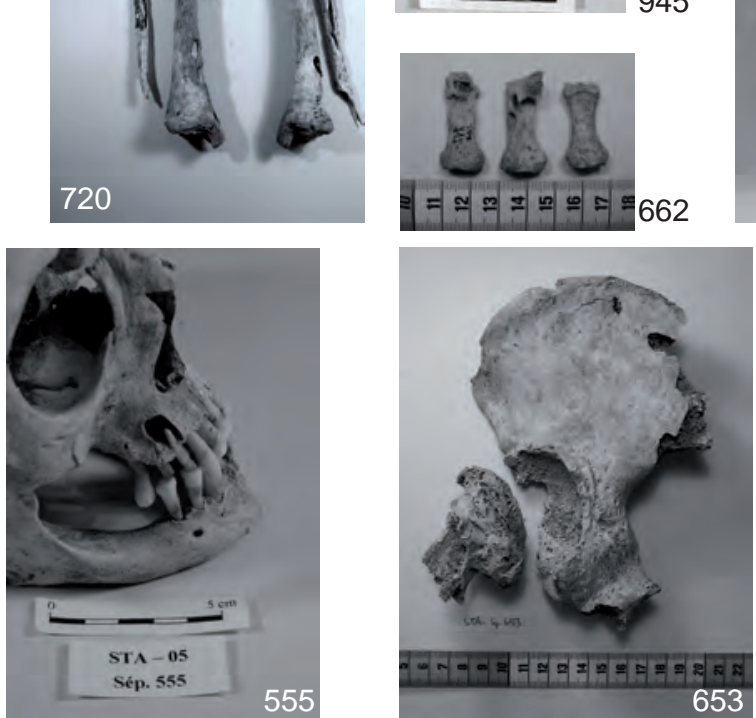

\section{2}

653
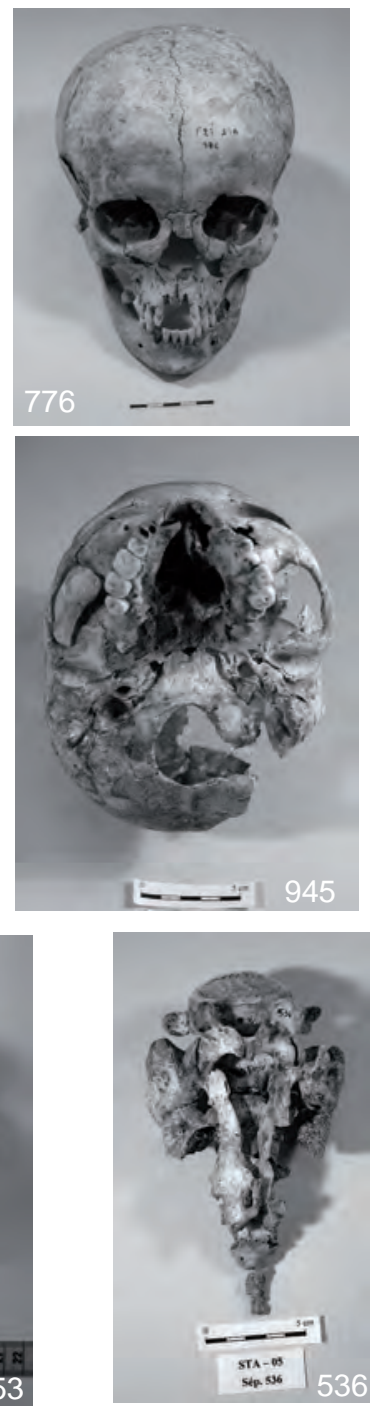

cette époque par les sources.

Les résultats de l'étude menée à Aizier tendent à montrer que la population qui y vivait était pauvre, mais que le mode de vie ne devait guère différer de la vie à la campagne : le mobilier, l'architecture des bâtiments, leurs dimensions et leur aménagement, reflètent les techniques et usages de l'époque, sans que l'on ait pu mettre en évidence une quelconque spécificité liée au statut particulier du site. Les résultats préliminaires de l'analyse anthropologique montrent qu'il n'y a pas de sélection, sur critère sexuel, des malades accueillis dans cette léproserie, mais qu'une sélection liée à l'âge existe. Les inhumés sont en effet principalement des sujets adultes, majoritairement jeunes. Même si une quinzaine d'immatures et d'adolescents ainsi que quatre périnatals ont été décomptés parmi les défunts, la population d'Aizier est loin de correspondre aux normes d'une population naturelle. L'étude menée permet
Fig. 17. Exemples d'atteintes pathologiques caractéristiques de la lèpre lépromateuse (clichés: C. Niel). 
également de mettre en valeur les soins apportés aux malades. Certains d'entre eux, parvenus au stade ultime de leur maladie, présentent un état sanitaire déplorable, notamment dans les régions rhino-maxillaire et bucco-dentaire. Toutefois, l'importance des atteintes montre que ces individus ont bénéficié d'une prise en charge sociale de leur maladie et d'une assistance jusqu'à leur décès et même au-delà avec une inhumation dans le cimetière communautaire. Ces premiers résultats nous donnent donc un éclairage privilégié sur la vie à la campagne entre le $\mathrm{XII}^{\mathrm{e}}$ et le XVI ${ }^{\mathrm{e}}$ siècle, plus généralement plutôt documentée par les fouilles de villages, de sites d'élites religieuses civiles ou militaires et plus rarement par des habitats ruraux isolés.

\section{Bibliographie}

BÉRIAC F. (1988) - Histoire des lépreux au Moyen âge, une société d'exclus. Paris, Imago.

Cardon T. (2010) - Étude archéo-numismatique du dépôt monétaire de la chapelle Saint-Thomas d'Aizier (Eure). Rapport d'étude, service régional de l'archéologie de Haute-Normandie.

Cardon T. (à paraître) - Le trésor d'Aizier : composition, structure et contexte archéologique. Numismatique et Normandie. Actes du colloque de Bayeux, Hollard D., Jérôme Jambu J. (dir.). Recherches et Travaux de la Séna, no 4.

CARdon T. (à paraître a) - Le trésor d'Aizier : composition et circulation monétaire. Thésauriser au XVe siècle. Paris, BNF (Trésors monétaires).

Duval P.-C. (1980) - Le réseau routier antique en Haute-Normandie (Nord de la Seine). Mémoire de maîtrise d'histoire, Éditions du Groupe archéologique du Val de Seine.

Duval P.-C. (1986) - Les routes anciennes de la Basse-Seine.

Fauroux M. (1961) - Recueil des actes des ducs de Normandie (911-1066). Mémoires de la Société des Antiquaires de Normandie, t. $36, \mathrm{n}^{\circ} 92$, p. $79-80, \mathrm{n}^{\circ} 9$.

Groupe archéologique du Val de Seine (1988) - 12 ans de recherches en forêt de Brotonne, 1976-1988. Ouvrage multigraphié déposé à la maison du Parc naturel régional des Boucles de la Seine normande.

GuÉRIN T. (2005) - Le fragon, données archéobotaniques préliminaires. Aizier, Chapelle Saint-Thomas, fouille programmée d’une léproserie médiévale, Truc M.-C. (dir.). Rapport intermédiaire, p. 54-60.

Henrion F. (1998) - Coffrages de bois, coffrages de planches (notes sur l'utilisation du bois dans le maintien des terres et matériaux autour du cadavre, Rencontre autour du cercueil, Bulletin de liaison du GAAFIF, $\mathrm{n}^{\circ}$ spécial 2, p. 31-33.

Keyzer W. de (2000) - L'évolution interne des léproseries à la charnière des XII et XIII ${ }^{\mathrm{e}}$ siècles : le cas de l'évêché de Cambrai. Lépreux et sociabilité du Moyen Âge aux Temps modernes. Rouen, PUR (Cahiers du GRHIS, no 11), p. 14-15.

Legros R. (1984) - Rapport du sondage effectué du 7 au 15 juillet 1984 à la chapelle Saint-Thomas d'Aizier. Rapport de fouilles, Service régional de l'archéologie de Haute-Normandie.

Lequoy M.-C. (1995) - Forêt de Brotonne, Aizier (Eure), notices 62-63. De la Gaule à la Normandie. Musées départementaux de la Seine-Maritime, p. 115.

Maneuvrier C. (2000) - Paysages et sociétés rurales au Moyen Âge : le Pays d'Auge jusqu'à la fin du XIIr siècle. Caen, thèse de doctorat.

Penna B. (1999) - Les données de l'histoire. Aizier, Chapelle Saint-Thomas, M.-C. Truc et B. Penna. Rapport de fouilles archéologiques programmées, Service régional de l'archéologie de Haute-Normandie, p. 23-28.

Rateau R. et Pinet J. (1870) - Histoire et géographie de l'Eure. Réimpression : Éditions de la Grande Fontaine.

Tabuteau B. (1995) - Une léproserie normande au Moyen Âge. Le prieuré de Saint-Nicolas d'Évreux du XII ${ }^{e}$ au XVI ${ }^{e}$ siècle. Histoire et corpus des sources. Thèse de doctorat, université de Rouen, J.-P. Leguay (dir.), 5 vol.

Truc M.-C., Niel C., Penna B. et Yvernault F. (2007) - La chapelle Saint-Thomas d'Aizier, premiers résultats de six années de fouille programmée. Études des lépreux et léproseries au Moyen Âge dans le nord de la France, Histoire, Archéologie, patrimoine, B. Tabuteau (dir.), Histoire médiévale et Archéologie, vol. 20, CAHMER, Laboratoire d'archéologie et d'histoire, université de Picardie - Jules-Verne, p. 47-109.

Uhrmacher M. (2000) - Leprosorien in Mittelalter und früher Zeit. Geschichtlicher Atlas der Rheinlande, Beiheft VIII-5, Köln, p. 21.

Yvernault F. (2005) - Nouvelles données historiques. Aizier, Chapelle Saint-Thomas, fouille programmée d'une léproserie médiévale, Truc M.-C. (dir.). Rapport intermédiaire, p. 15-31. 


\section{JOURNÉES}

\section{$\mathrm{DE}$}

\section{ARCHEOLOGIQUES}

\section{HAUTE-NORMANDIE}

J.-P. Watté Les occupations Mésolithique et Cerny de type «Barbuise » du site de La Mare du Hom, à Quittebeuf (Eure) • F. KLiesch Nonancourt "La Potinière » (Eure). Une partie de la nécropole à enclos circulaires de la fin de l'âge du bronze ancien au début de l'âge du bronze moyen - C. Moreau et F. Granier Val-de-Reuil (Eure), Le Chemin aux Errants, une occupation rurale

Évreux,

6-8 mai

2011 du premier et second âge du fer à la confluence de l'Eure et de la Seine • D. Lukas, H. Delnef et L. Leconte Les établissements laténien et gallo-romain de Heudebouville (Eure), Premier bilan des fouilles menées en 2009 et 2010 à l'« Écoparc 2 • P. Wech Beuzeville (Eure), une modeste occupation rurale gallo-romaine en Pays d'Auge - J. Mouchard Le port romain d'Aizier (Eure) : principaux résultats de la campagne 2010 • F. FERreIra Le théâtre antique du sanctuaire du Vieil-Évreux (Eure) - M. Michel Authevernes « Les Mureaux » (Eure) : le sanctuaire antique, approche chronologique et pratiques cultuelles $\bullet$ S. BERTAudière, S. Cormier et L. Guyard Le grand sanctuaire central du Vieil-Évreux (Eure), résultats de la campagne 2010 - C. Hartz Les habitations et la trame urbaine du Vieil-Évreux (Eure), résultats de la campagne 2010 • F. Ferreira Le théâtre antique du sanctuaire du Vieil-Évreux (Eure) - V. Mutarelli Le théâtre romain de Lillebonne (Seine-Maritime), chantier de fouilles 2010 - É. Mantel et S. Dubois Premier éclairage sur l'agglomération antique de Briga (Eu, Bois l'Abbé, Seine-Maritime), fenêtres ouvertes sur un quartier d'habitation au nord-est du centre monumental - F. Pilon Découvertes monétaires en contexte funéraire gallo-romain en Haute-Normandie, bilan de quelques études récentes $\bullet$ F. CARRÉ Louviers (Eure), rue du Mûrier : de nouvelles informations sur le cimetière du haut Moyen Âge • G. Deshayes, B. Lepeuple et D. Théolas Les occupations médiévales autour de Gisors (Eure), du haut Moyen Âge à la fortification Plantagenêt • N. Roudié et D. Jouneau Premiers vestiges archéologiques du château médiéval du Vaudreuil (Eure) - A. Painchault Gaillefontaine (Seine-Maritime), approche topographique d'une fortification du Pays de Bray • J.-B. Vincent Une approche archéologique globale des abbayes cisterciennes normandes : l'exemple de l'abbaye de Fontaine-Guérard • C. NieL et M.-C. Truc La chapelle Saint-Thomas d'Aizier (Eure) : bilan de douze années de fouille programmée • D. Piтte Les villes et agglomérations médiévales de Haute-Normandie révélées par leurs caves - J.-L. CAmuset Ivry-la-Bataille (Eure), la " Grotte du sabotier », premiers résultats d'une fouille programmée • V. Le Borgne, J.-N. Le Borgne et G. Dumondelle L'archéologie aérienne dans le département de l'Eure en 2010.

\section{PUBLICATIONS DES UNIVERSTÉS DE ROUEN ET DU HAVRE}

ISBN : 978-2-87775-559-7
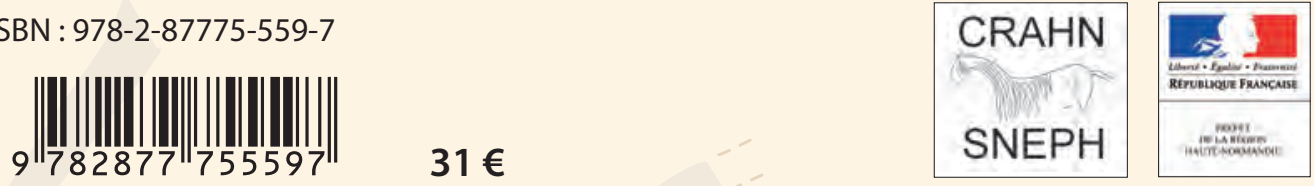

U DUNERESTIEE 